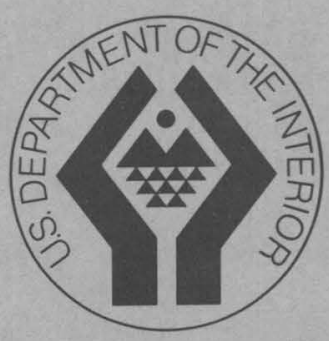

Index of Surface-Water Records to September 30, 1967

Hawaii and Other Pacific Areas

GEOLOGICAL SURVEY CIRCULAR 586 



\title{
Index of Surface-Water Records tc September 30, 1967 Hawaii and Other Pacific Areas
}

\author{
By H. P. Eisenhuth
}

GEOLOGICAL SURVEY CIRCULAR 586

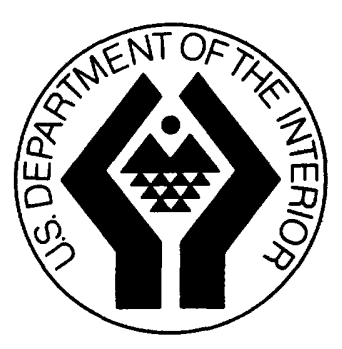


United States Department of the Interior STEWART L. UDALL, Secretary

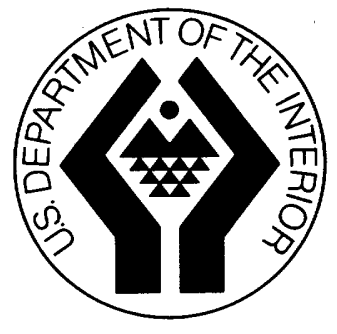

Geological Survey

William T. Pecora, Director

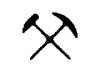




\title{
Index of Surface-Water Records to September 30, 1967
}

\author{
Hawaii and Other Pacific Areas
}

\author{
By H. P. Eisenhuth
}

\section{INTRODUCTION}

This report lists the streamflow stations in Hawaii and other Pacific areas for which records have been or are to be published in reports of the Geological Survey for periods through September 30, 1967. It supersedes Geological Survey Circular 515 .

Basic data on surface-water supply of the United States prior to 1961 have been published in an annual series of water-supply papers consisting of several volumes, one of which was for Hawaii. Water-Supply Paper 1751, entitled "Surface Water Supply of Mariana, Caroline and Samoa Islands through June 1960" contains data for other Pacific areas.

Beginning in 1961, the annual series of water-supply papers on surface-water supply was changed to a 5-year series. Records for the period 1961-65 will be published in a water-supply paper entitled "Surface Water Supply of Hawaii and other Pacific Areas." In order to meet interim requirements, beginning July 1, 1960, the Hawaii district issues reports annually containing streamflow records for Hawaii and other Pacific areas.

In addition to the continuous-record gaging stations, this index includes crest-stage and low-flow partial-record stations. A continuous-record station is a gaging station on a stream or reservoir for which the discharge, stage, or contents is published on a daily, weekly, or monthly basis for a continuous period of time. A crest-stage partial-record station is a streamflow station for which only the annual maximum discharge is published over a period of years for use in floodflow analyses. A low-flow partial-record station is a streamflow station for which only discharge measurements made at base flow, when streamflow is primarily from ground-water storage, are published. Measurements are generally collected over a period of five years or more for use in low-flow analyses. Discharge measurements have been made at many sites and published as miscellaneous measurements. Such measurements are not included in this index except those made in earlier years at sites now classified as low-flow partial-record stations.

Explanation of reference marks is given at the end of the index.

An alphabetical list of streams is given at the end of this circular.

\section{DOWNSTREAM ORDER}

The stations in this index are listed in the downstream order used in the series of water-supply papers on surfacewater supply of Hawaii since 1951. Starting at the headwater of each stream all stations are listed in a downstream direction. Tributary streams are indicated by indention and are inserted between main-stem stations in the order in which they enter the main stream. To indicate the rank of any tributary on which a record is available and the stream to which it is immediately tributary, each indention in the listing of stations represents one rank. A stream name, only, is inserted where necessary for the purpose of showing the proper rank or order of tributaries.

\section{STATION NAMES}

Station names are given in their most recently published form. Parentheses around part of a station name indicate that the enclosed word or words were used in an earlier published name of the station or as an alternate name. Parenthetical explanations are also used to indicate that a stream of a different name is the head of the main stream by use of "(head of River)". River)" and that the name of the main stream has changed by use of "(continuation of 


\section{STATION NUMBER}

As an added means of identification, each continuous- and partial-record station has been assigned a station number. The numbers have been assigned in the same downstream order used in this index. In assigning station numbers, gaps were left to allow for new stations that may be established; hence the numbers are not consecutive. The first tro digits of the complete number for each station identify the area, they are 16 for all stations in this report. The number given consists of only the essential digits of the complete number. For example, for a station with the complete number $16-0950.00$, the station number shown in this index is 0950 .

\section{DRAINAGE AREA}

The drainage area, in square miles, is the latest figure published or otherwise available at this time.

\section{PERIODS OF RECORD}

Under "Periods of record" are three columns. The first column, "Daily or monthly figures," shows the periods of record for continuous-record gaging stations. The dates given are the calendar years in which records began or ended; breaks of less than a year are not shown. For example, if a record began in October 1923, ended in April 1932, began again in March 1933, and ended in September 1944, the period of record would be shown as 1923-44.

The second column, "Annual peaks," shows the period of record for crest-stage partial-record stations. The dates given are fiscal years for which the annual maximum is available. The fiscal year begins July 1 and ends Jure 30 . In listing the fiscal year, only one date is shown; for example, 1952 stands for the fiscal year July 1, 1951, to June $30,1952$.

The third column "Low-flow measurements," shows the period of record for low-flow partial-record stations, the dates given are the fiscal years in which base-flow measurements were made at low-flow partial-record stations: breaks of less than two years are not shown. Prior to the 1958 water year, such measurements were published in tables headed "Miscellaneous discharge measurements" or "Discharge measurements at sites other than gaging stations." Many discharge measurements have been made at miscellaneous sites which are not listed in this index because the data collected were not sufficient to qualify the site as a low-flow partial-record station.

A date followed by only a dash shows that the station was continued in operation beyond September 30,1967 . A date followed by a period indicates discontinuance. A date followed by a semicolon indicates a break in the collection of records. 
Periods of record

\section{HAWAII}

\begin{tabular}{|c|c|c|c|c|}
\hline \multirow{2}{*}{$\begin{array}{l}\text { Station } \\
\text { number }\end{array}$} & \multirow{2}{*}{$\begin{array}{l}\text { Drainage area } \\
\quad(\text { sq mi })\end{array}$} & \multicolumn{3}{|c|}{ Periods of record } \\
\hline & & $\begin{array}{c}\text { Daily or monthly figures } \\
\text { (calendar years) }\end{array}$ & $\begin{array}{l}\text { Annual peaks } \\
\text { (fiscal years) }\end{array}$ & $\begin{array}{l}\text { Low-flow measurements } \\
\text { (fiscal years) }\end{array}$ \\
\hline \multicolumn{5}{|l|}{$16-$} \\
\hline 0100 & a4.1 & $1909-16 ; 1919-$ & & \\
\hline 0110 & al. 7 & $1909-10 ; 1912 * ; 1919-25 \ldots \ldots \ldots$ & ........... & 1914-18;1961- \\
\hline 0120 & 1.17 & $1919-25 \ldots \ldots \ldots \ldots \ldots \ldots \ldots$ & $\cdots \cdots \cdots \cdots \cdots$ & $1912-17 ; 1961-$ \\
\hline 0130 & al. 6 & $1920-26 ; 1936-$ & & \\
\hline 0140 & - & $1926-$ & & \\
\hline $\begin{array}{l}0150 \\
0160\end{array}$ & $\begin{array}{l}22.2 \\
20.0\end{array}$ & $\begin{array}{l}1909-10 ; 1911-17 \mathrm{~b} \text {. } \\
1916 ; 1917-18 ; 1925-\end{array}$ & & \\
\hline 0170 & 3.33 & $1919-32 ; 1954-$ & & \\
\hline 0180 & 11.7 & $1916-18 \ldots \ldots \ldots \ldots \ldots \ldots \ldots$ & $1962-\ldots \ldots \ldots$ & $1916 ; 1920-21 ; 1954-55$; \\
\hline 0190 & 22.5 & $1920-32 ; 1952-$ & & \\
\hline $\begin{array}{l}0200 \\
0210\end{array}$ & $\begin{array}{l}23.5 \\
8.75\end{array}$ & $\begin{array}{l}1910-16 . \\
1916-21\end{array}$ & & \\
\hline 0220 & - & $1908-$ & & \\
\hline 0240 & - & $1910-12 *$. & & \\
\hline $\begin{array}{l}0250 \\
0270\end{array}$ & $\overline{-}$ & $\begin{array}{l}1910-12^{*} \\
1908-14 ; 1916-34 .\end{array}$ & & \\
\hline 0280 & 45.0 & $1921-55 \ldots \ldots \ldots \ldots \ldots \ldots$ & 1956- & \\
\hline 0290 & - & $1911-13 ; 1916-21$. & & \\
\hline 0295 & 4.77 & ${ }^{1960-} \ldots \ldots \ldots \ldots \ldots \ldots$ & $1962-64 \ldots \ldots \ldots$ & $1962-$ \\
\hline 0310 & 57.8 & $19.10-18 ; 1943-$ & & \\
\hline 0320 & - & $1910-17$. & & \\
\hline 0330 & - & $1912-17$ & & \\
\hline $\begin{array}{l}0340 \\
0350\end{array}$ & $\begin{array}{r}24.9 \\
\text { a. } 6\end{array}$ & $\begin{array}{l}1915-16 . \\
1913 .\end{array}$ & & \\
\hline 0360 & a.25 & $1943-$ & & \\
\hline 0370 & $2025^{-}$ & $1911-13$ & & \\
\hline 0371 & & $1911-17$ & & \\
\hline 0380 & 88.0 & $1943-61 * c \ldots \ldots \ldots \ldots \ldots \ldots$ & $1943-$ & \\
\hline $\begin{array}{l}0390 \\
0410\end{array}$ & - & $1911-15$. & & \\
\hline 0410 & - & $1911-12^{*} \cdot$ & & \\
\hline 0420 & - & $1911-12 ; 1914-15$. & & \\
\hline 0430 & - & $1930-38$ & & \\
\hline $\begin{array}{l}0440 \\
0450\end{array}$ & - & $\begin{array}{l}1910-21 ; 1927-49 . \\
1929-32 .\end{array}$ & & \\
\hline 0460 & - & $1912-13 ; 1915-17$ & & \\
\hline $\begin{array}{l}0470 \\
0480\end{array}$ & $\begin{array}{r}12.6 \\
5.44\end{array}$ & $1910-16$ & & \\
\hline 0490 & 18.8 & $1917-21 ; 1926-$ & & \\
\hline 0500 & - & $1929-32$ & & \\
\hline 0510 & 20.5 & $1929-32$. & & \\
\hline 0520 & 26.9 & $1949-60^{*} c \ldots \ldots \ldots \ldots \ldots \ldots$ & $1961-$ & \\
\hline
\end{tabular}

Kawalkoi Stream (head of Waimea River) near Waimea

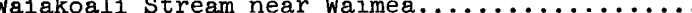
Kauaikinana Stream near Waimea.

Poomau Stream (continuation of Kawaikoi stream Mohihi Stream at altitude 3,420 ft $(3,500 \mathrm{ft})$ near Waimea (near Waimea).

okee ditch near waimea......... Mohihi Stream near Waimea....

Wa1mea River at altitude $840 \mathrm{ft}$ ( $890 \mathrm{ft})$, near Stream near Waimea)

Koale Stream at altitude $3,770 \mathrm{ft}(3,700 \mathrm{ft})$, near Waimea.

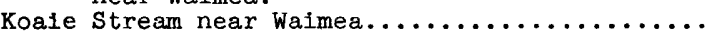

Waialae Stream (River) at altitude 3,820 ft $(3,700 \mathrm{ft})$, near Waimea.

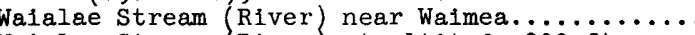

Walalae Stream (River) at altitude $800 \mathrm{ft}$, near Waimea.

Kekaha ditch at Camp 1 (at intake) (at Flume No. 4), near Waimea (near Waimea).

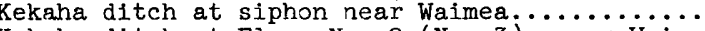
Kekaha ditch at Flume No. 2 (No. 3), near Waime Kekaha ditch below (at) tunnel 12 , near Waimea.

Waimea River below Kekaha ditch intake, near Waimea.

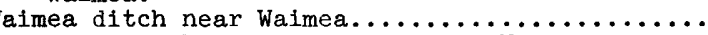
Waimea ditch below wasteway, near Waimea........

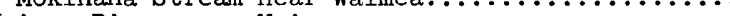
Olokele River (head of Makaweli River):

lokele ditch at tunnel 12 , Olokele ditch at tunnel 12, near Makaweli......

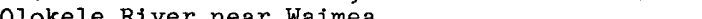
Halekua Stream near Waimea.

Makaweli River near Waimea. near Waimea (near Waimea).

Waimea River at Waimea. . . . . . . . . ...........

Hanapepe River:

Hiloa ditch (at Hanapepe Falls) near Eleele..... East Branch Hanapepe River at Hanapepe Falls, near Eleele.

Hanapepe ditch at Hanapepe Falls, near Fleele... Hanepe ditch below intake, near Eleele.......

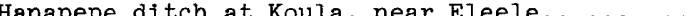
Hanapepe ditch below makai siphon, near Eleele. Hanape ditch at weir, near Hanapepe.......... Tanapepe River at Koulo near Eleele........ Manuahi Stream at Koula near Eleele.

Hanapepe River below Manuahi Stream (at Koula) near Eleele.

"G.

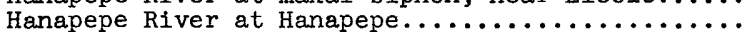




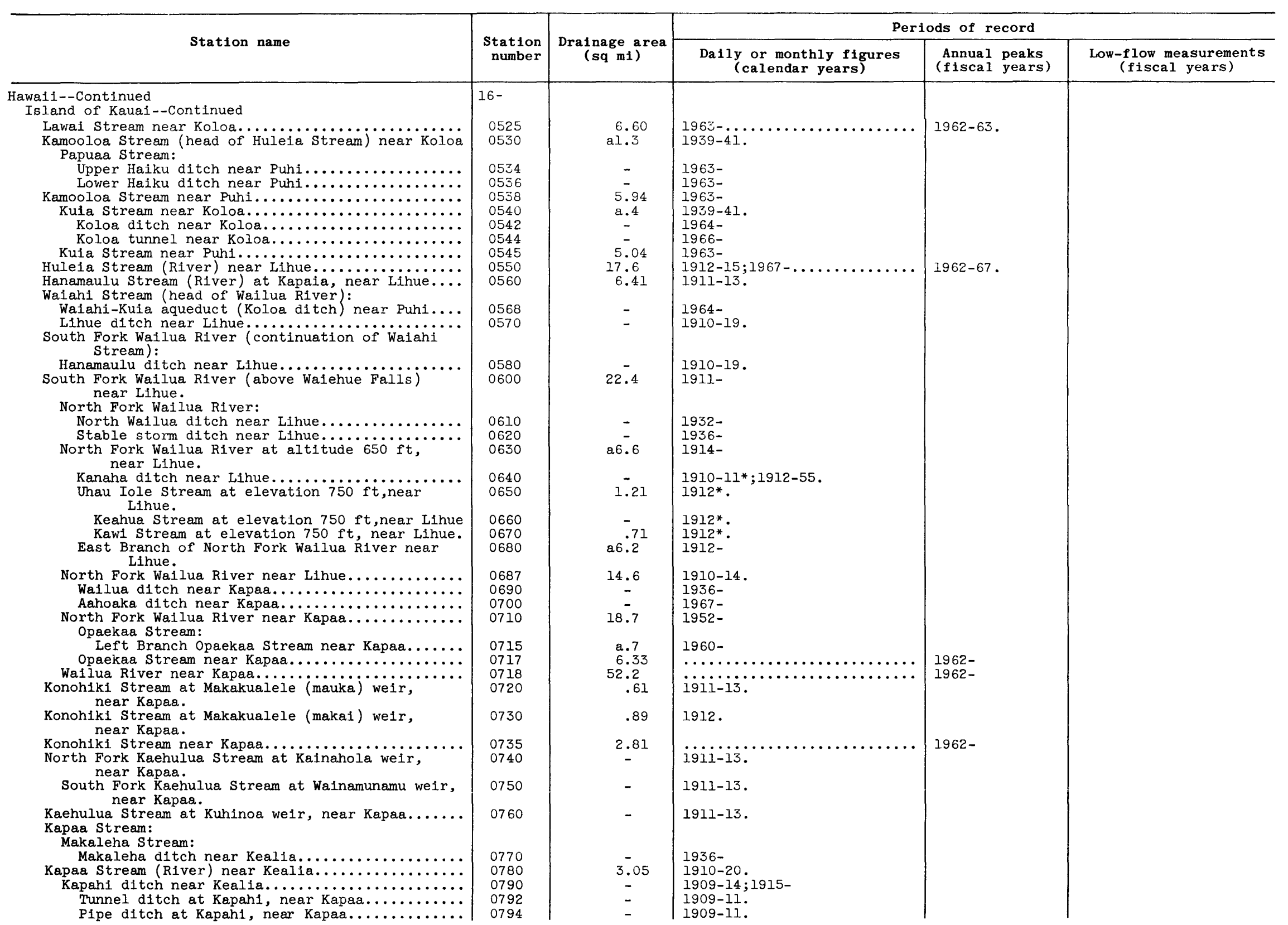


Kapaa ditch at Kapah1, near Kapaa.

\begin{tabular}{|c|c|c|c|}
\hline $\begin{array}{l}0796 \\
0800\end{array}$ & a3.3 & $\begin{array}{l}1909-11 . \\
1936-\end{array}$ & \\
\hline $\begin{array}{l}0810 \\
0812\end{array}$ & -42 & $\begin{array}{l}1911-13 . \\
\ldots \ldots \ldots \ldots \ldots \ldots \ldots \ldots\end{array}$ & 1964- \\
\hline $\begin{array}{l}0820 \\
0845 \\
0850 \\
0859 \\
0860\end{array}$ & $\begin{array}{c}- \\
13.2 \\
\therefore \\
-\end{array}$ & 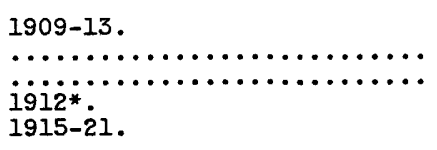 & $\begin{array}{l}1962- \\
1964-\end{array}$ \\
\hline $\begin{array}{l}0870 \\
0880\end{array}$ & $\overline{-}$ & $\begin{array}{l}1936- \\
1921-\end{array}$ & \\
\hline 0890 & a5.5 & $1910 \mathrm{~b} ; 1912-$ & \\
\hline 0900 & - & $1909-14$ & \\
\hline $\begin{array}{l}0910 \\
0920\end{array}$ & $\overline{-}$ & $\begin{array}{l}1936- \\
1909-10 .\end{array}$ & \\
\hline 0930 & 25.5 & $1910-11 ; 1912 *$ & \\
\hline 0932 & 9.89 & $1964-\ldots \ldots \ldots \ldots \ldots \ldots \ldots$ & $1962-65$ \\
\hline $\begin{array}{l}0942 \\
0950 \\
0952\end{array}$ & $\overline{-}$ & $\begin{array}{l}1932- \\
1932- \\
1956-\end{array}$ & \\
\hline $\begin{array}{l}0959 \\
0960 \\
0970 \\
0973 \\
0975\end{array}$ & $\begin{array}{l}- \\
- \\
\text { al. } \\
.15 \\
\text { al. }\end{array}$ & $\begin{array}{l}1960- \\
1934- \\
1957- \\
1922-25 . \\
1957-\end{array}$ & \\
\hline $\begin{array}{l}0979 \\
0980 \\
0990 \\
0995\end{array}$ & $\begin{array}{l}.87 \\
3.34 \\
3.92 \\
-\end{array}$ & $\begin{array}{l}\text { i9i4-23. } \cdots \cdots \ldots \ldots \ldots \ldots \ldots \ldots \\
\text { 1912-13. } \\
1956-62 .\end{array}$ & $1964-$ \\
\hline $\begin{array}{l}1000 \\
1010 \\
1020 \\
1030 \\
1040\end{array}$ & $\begin{array}{l}- \\
27.4 \\
- \\
19.1\end{array}$ & $\begin{array}{l}1932- \\
1914-55 . \\
1912-19 ; \\
1912-19 ; 1962-\ldots \ldots \ldots \ldots \ldots \ldots \\
1912-13 ; 1916-19 .\end{array}$ & 1962. \\
\hline $\begin{array}{l}1042 \\
1050 \\
1060 \\
1070 \\
1080 \\
1090\end{array}$ & $\begin{array}{l}21.0 \\
0.1 .5 \\
0.5 \\
- \\
10.2 \\
11.4\end{array}$ & $\begin{array}{l}19314-32 ; \ldots \ldots \ldots \ldots \ldots \ldots \ldots \\
1914-17 ; 1920-33 . \\
1912 * \text {. } \\
1952-56 ; 1957- \\
1914-16 .\end{array}$ & 1963- \\
\hline $\begin{array}{l}1100 \\
1110 \\
1120 \\
1130 \\
1150 \\
1160 \\
1170 \\
1300\end{array}$ & $\begin{array}{l}- \\
- \\
14.7 \\
\text { a2.6 } \\
\text { al. } \\
\text { al. } 6 \\
3.77 \\
7.79\end{array}$ & $\begin{array}{l}1910-16 . \\
1911 . \\
1911 . \\
1912-16 . \\
1931-52 . \\
1931-52 . \\
1931-55 . \\
1953-\ldots \ldots \ldots \ldots \ldots \ldots \ldots \ldots \ldots\end{array}$ & $19.52-53$ \\
\hline
\end{tabular}
.

Akulikuli Spring at welr, near Kapaa...........

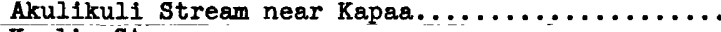
ealia stream:

(at weir) near Keall

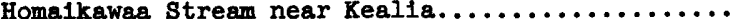
Anahola River at elevation 1,140 ft, near Keal1a..

Anahola ditch above wasteway (Kaneha Reservolr), near Kealia.

Anahola ditch wasteway near Kealla........... Anahola ditch above Kaneha Reservoir, near

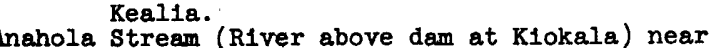
Keal1a.

Lower Anahola (Anahola) ditch at Klokala, near Kealia.

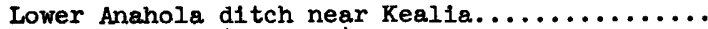

Lower Anahola (Anahola) ditch at makai weir, near Kealia.

Anahola Stream (River above dam) at Kiokala Dam, near Kealia.

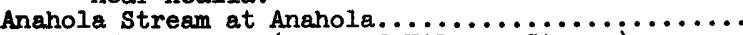

Puu Ka Ele Stream (head of Kilauea Stream)

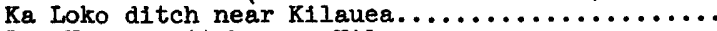

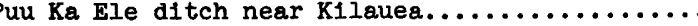
Ross ditch near Kllauea..................

Kalihiwas ditch above wasteway, near Kilauea. Kalihiwal ditch above wasteway, near Kilauea.. Poliniwal ditch near Kilauea.

Halaunu stream near Kilauea.................. Halaulan stream at altitude $400 \mathrm{f} t$, near Kilauea.

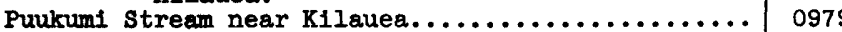

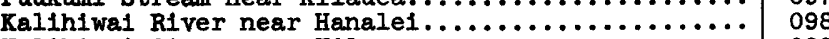

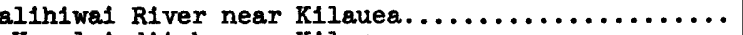

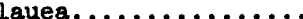

Hanalei River:

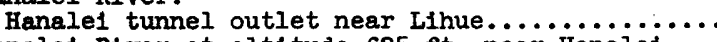

Chin ditch at altitude 625 1 , near Hanale1...

Hanele1 River near Hanglei

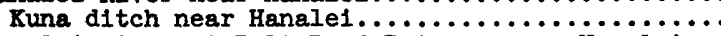

Hanale1 River at Belt Road Bridge, near Hanale1..

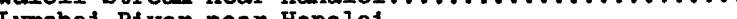

Tumat Rtver near Wainitha 0.0 .0 .0 .0 .0 .0 .0 .0 .0$.

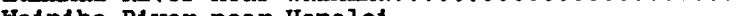

Wainthe Biver above intake near Hanalei (near Wainina).

Waintha Canal at intake, near Waintha...........

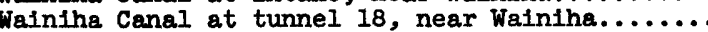

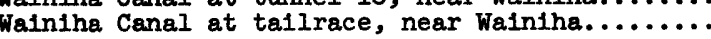

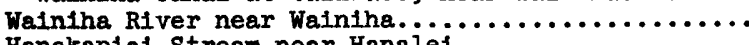

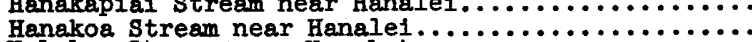

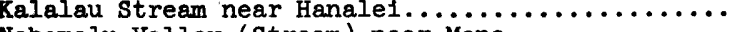

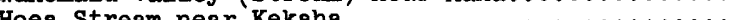




\begin{tabular}{|c|c|c|c|c|c|}
\hline \multirow{2}{*}{ Station name } & \multirow[b]{2}{*}{$\begin{array}{r}\text { Station } \\
\text { number }\end{array}$} & \multirow[b]{2}{*}{$\begin{array}{l}\text { Drainage area } \\
\quad(\mathrm{sq} \mathbf{m i})\end{array}$} & \multicolumn{3}{|c|}{ Periods of record } \\
\hline & & & $\begin{array}{l}\text { Daily or monthly figures } \\
\text { (calendar years) }\end{array}$ & $\begin{array}{l}\text { Annual peaks } \\
\text { (fiscal years) }\end{array}$ & $\begin{array}{l}\text { Low-flow measurements } \\
\text { (fiscal years) }\end{array}$ \\
\hline 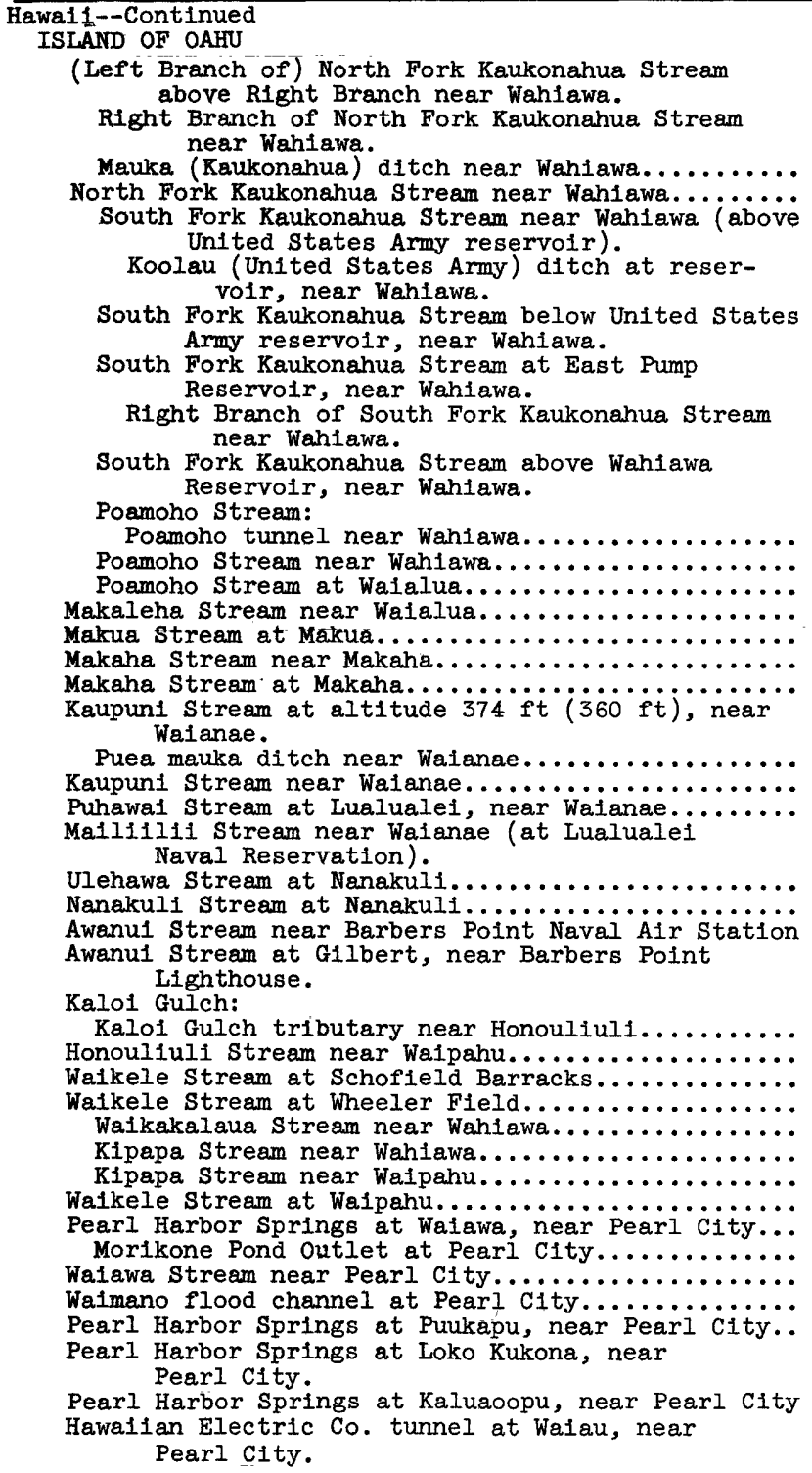 & \begin{tabular}{|l|}
$16-$ \\
2000 \\
2010 \\
2030 \\
2040 \\
2060 \\
2065 \\
2070 \\
2080 \\
2085 \\
2090 \\
\\
2109 \\
2110 \\
2112 \\
2113 \\
2115 \\
2116 \\
2117 \\
2118 \\
2118.5 \\
2119 \\
2120 \\
2122 \\
2122.5 \\
2123 \\
2124 \\
2124.01 \\
\\
2124.5 \\
2125 \\
2126 \\
2126.01 \\
2127 \\
2128 \\
2129 \\
2130 \\
2140 \\
2150 \\
2160 \\
2165 \\
2170 \\
2180 \\
2185 \\
2190 \\
\end{tabular} & $\begin{array}{c}1.38 \\
1.20 \\
- \\
4.86 \\
1.93 \\
- \\
24.0 \\
4.04 \\
.86 \\
5.26 \\
\\
- \\
1.79 \\
10.9 \\
4.15 \\
4.13 \\
2.31 \\
5.25 \\
3.58 \\
- \\
4.39 \\
2.6 \\
1.51 \\
4.51 \\
3.98 \\
1.16 \\
2.50 \\
1.70 \\
11.0 \\
5.05 \\
6.35 \\
6.96 \\
4.29 \\
14.8 \\
45.7 \\
- \\
- \\
26.4 \\
2.63 \\
- \\
- \\
- \\
-\end{array}$ & 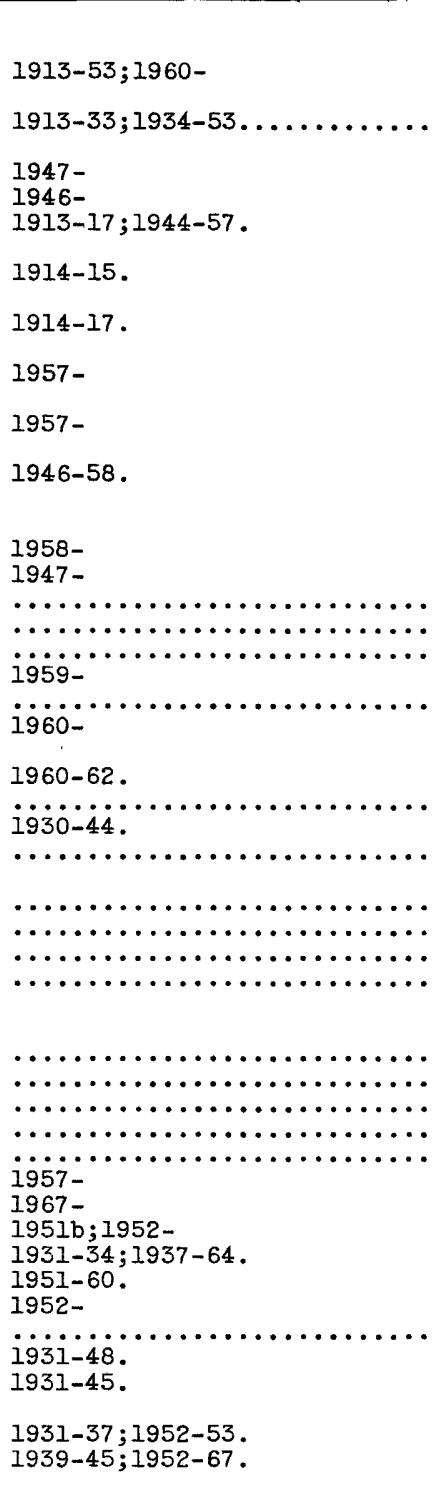 & $\begin{array}{l}1967- \\
1958- \\
1958- \\
1967- \\
\\
1958-60 . \\
1958- \\
1967- \\
1967- \\
1958 . \\
1959-64 . \\
1967- \\
1955- \\
1958 . \\
1958- \\
1958- \\
\end{array}$ & $1960-62$; 1966- \\
\hline
\end{tabular}




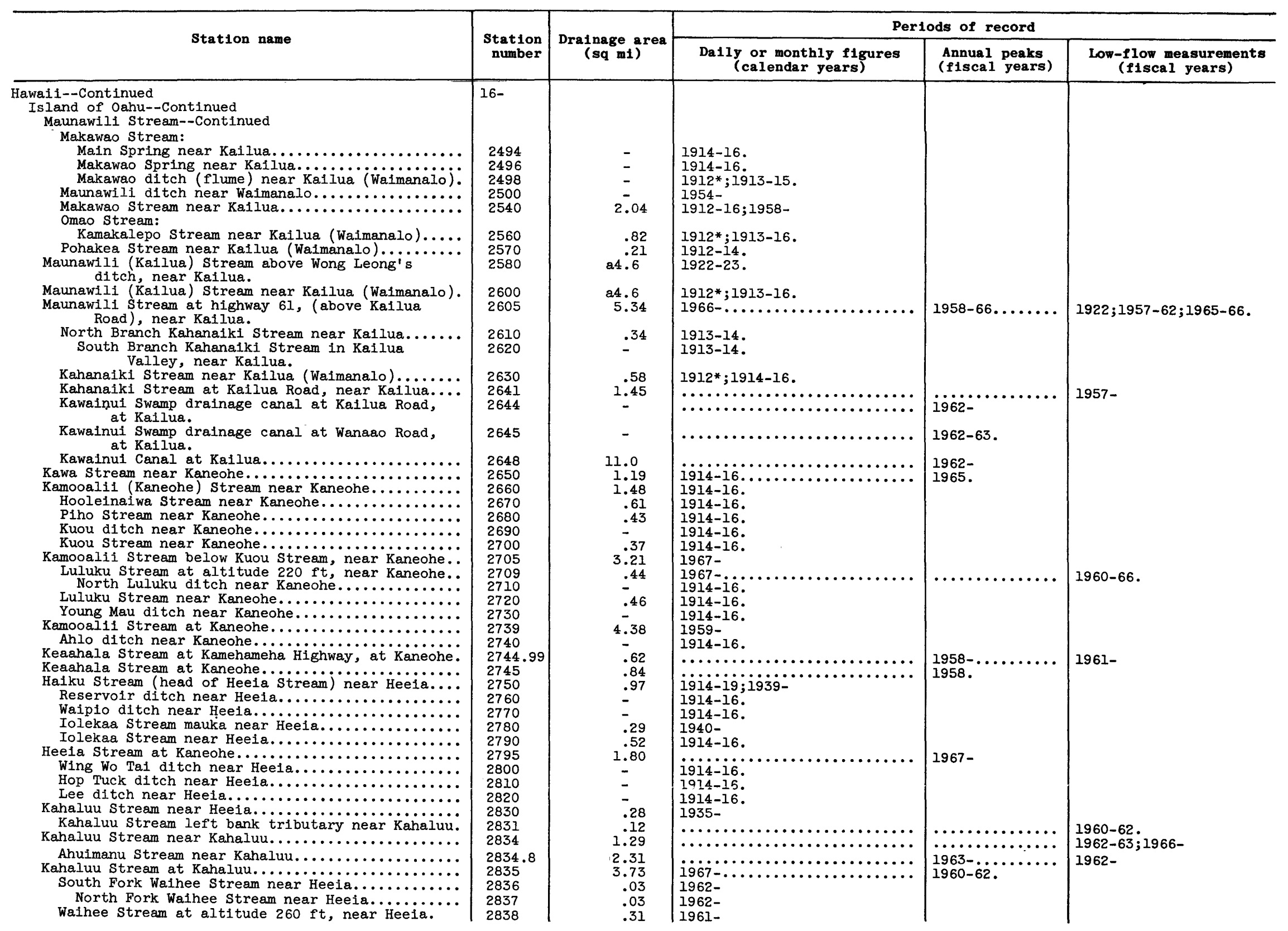


Wathee Stream near Heela

Wathee tunnel at Waianu, near Waiahole..... Walahole tunnel wasteway at intake 31 , near

$$
\text { Waiahole. }
$$

Waiahole tunnel at north portal, near Walahole.... Waiahole tunnel at adit 8 , near Waipahu..........

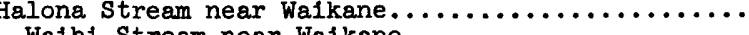
Waiahole stream below powerhouse, near Waiahole.... Waiahole stream at altitude $250 \mathrm{ft}$, near Walahole.

Waiahole Stream near Waiahole (at Manianiaula,

$$
\text { near Waikane). }
$$

Waianu Stream near Waikane.

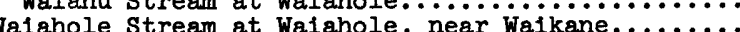
Waikane Stream at altitude $75 \mathrm{ft}$, near Walkane...

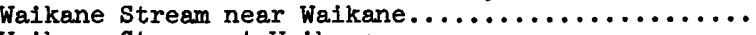
Walkane Stream at Waikane.

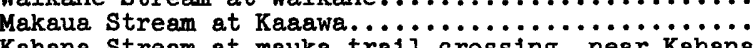
Kahana Stream at mauka trail crossing, near Kahana Kahana Stream near Kahana. $¥ \ldots \ldots \ldots \ldots \ldots \ldots \ldots$ Kawa (East Branch Kahana) Stream near Kahana....

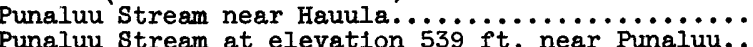

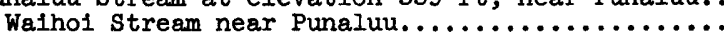
Punaluu Stream at elevation $250 \mathrm{ft}$, near Punaluu..

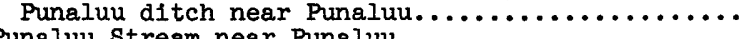

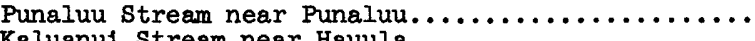

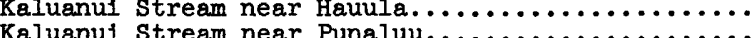
Kaluanul stream at altitude $30 \ldots \ldots \ldots \ldots \ldots \ldots \ldots$

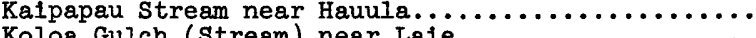

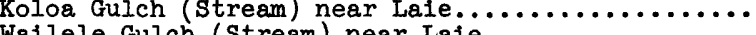

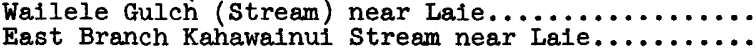

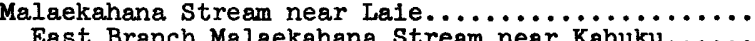
East Branch Malaekahana Stream near Kahuku...... Middle Branch Malaekahana Stream near Kahuku....

Malaekahana Stream at altitude $70 \mathrm{ft}$, near Kahuku.
Malaekahana Stream at altitude $30 \mathrm{ft}$, near Kahuku.

Malaekahana Stream at altitude $30 \mathrm{ft}$, near Kahuku.

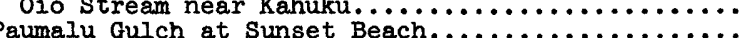

Paumalu Gulch

Kamananui Stream at Pupukea Military Road, near Maunawa1.

awaikoele Stream:

Kawaikoele Stream tributary near Maunawai...

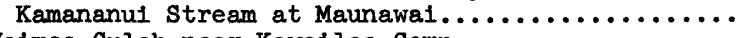

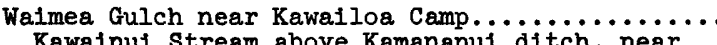
Wahiawa.

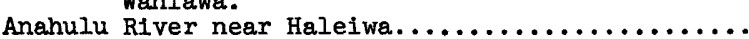
Anahulu River tributary near Haleiwa...........

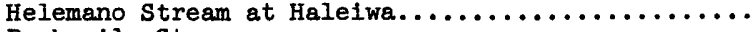
Paukauila Stream:

Opaeula Stream near Wahtawa. ................. 3450 opaeula Stream near Haleiwa................. 3500 ISLAND OF MOLOKAI

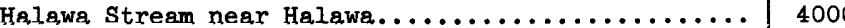
Papalaua stream near Pukoo.................... 4010

Pulena Stream (head of Wallau Stream) near Wailau. 4020 Walakeakua stream near Wallau............... 4030

$1960-66$

$1961-$

$1958-60$.

$1958-62$

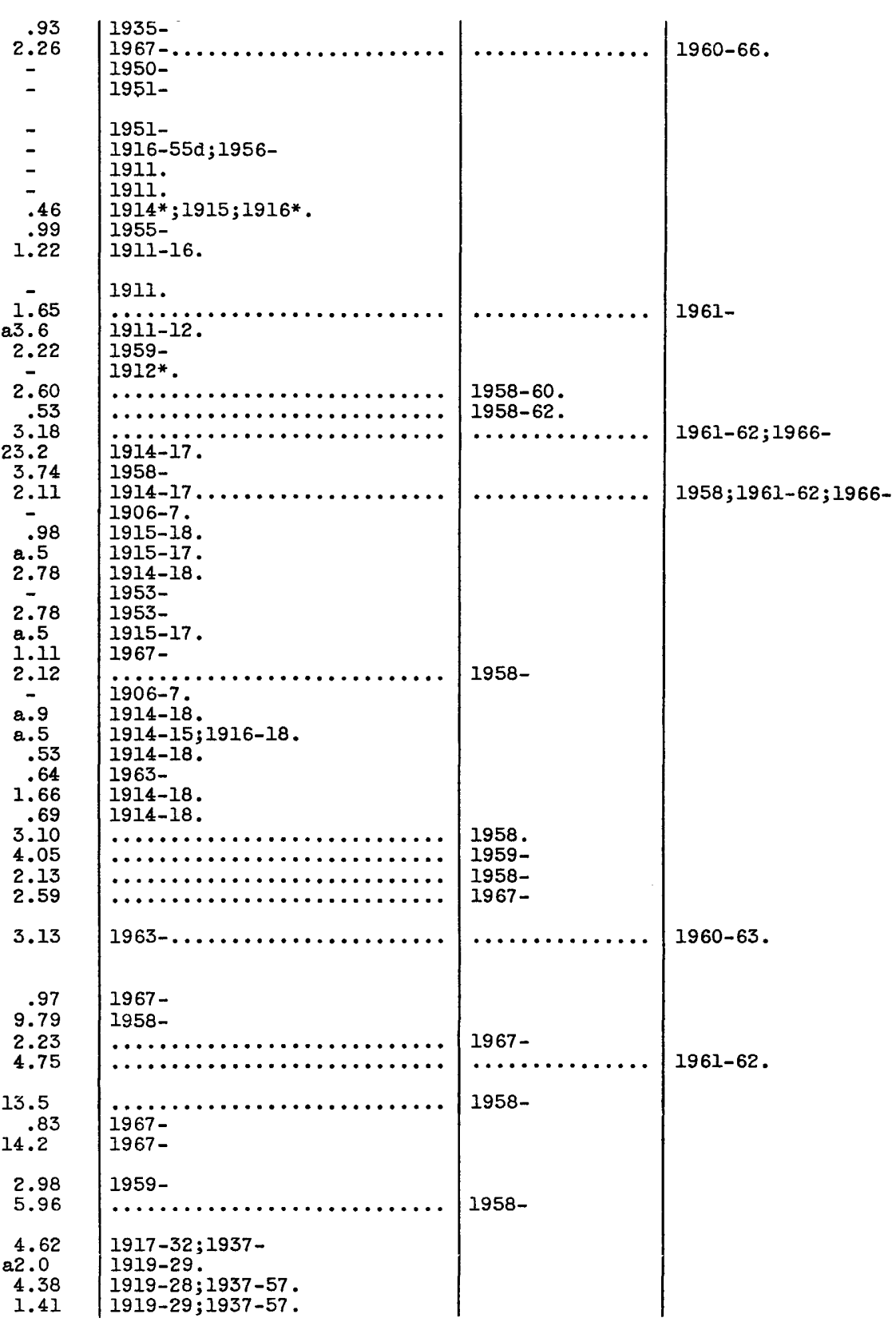




\begin{tabular}{|c|c|c|c|c|c|}
\hline \multirow{2}{*}{ Station name } & \multirow{2}{*}{$\begin{array}{r}\text { Station } \\
\text { number }\end{array}$} & \multirow{2}{*}{$\begin{array}{l}\text { Drainage area } \\
(\mathrm{sq} \mathrm{mi})\end{array}$} & \multicolumn{3}{|c|}{ Periods of record } \\
\hline & & & $\begin{array}{l}\text { Daily or monthly figures } \\
\text { (calendar years) }\end{array}$ & $\begin{array}{c}\text { Annual peaks } \\
\text { (fiscal years) }\end{array}$ & $\begin{array}{l}\text { Low-flow measurements } \\
\text { (fiscal years) }\end{array}$ \\
\hline 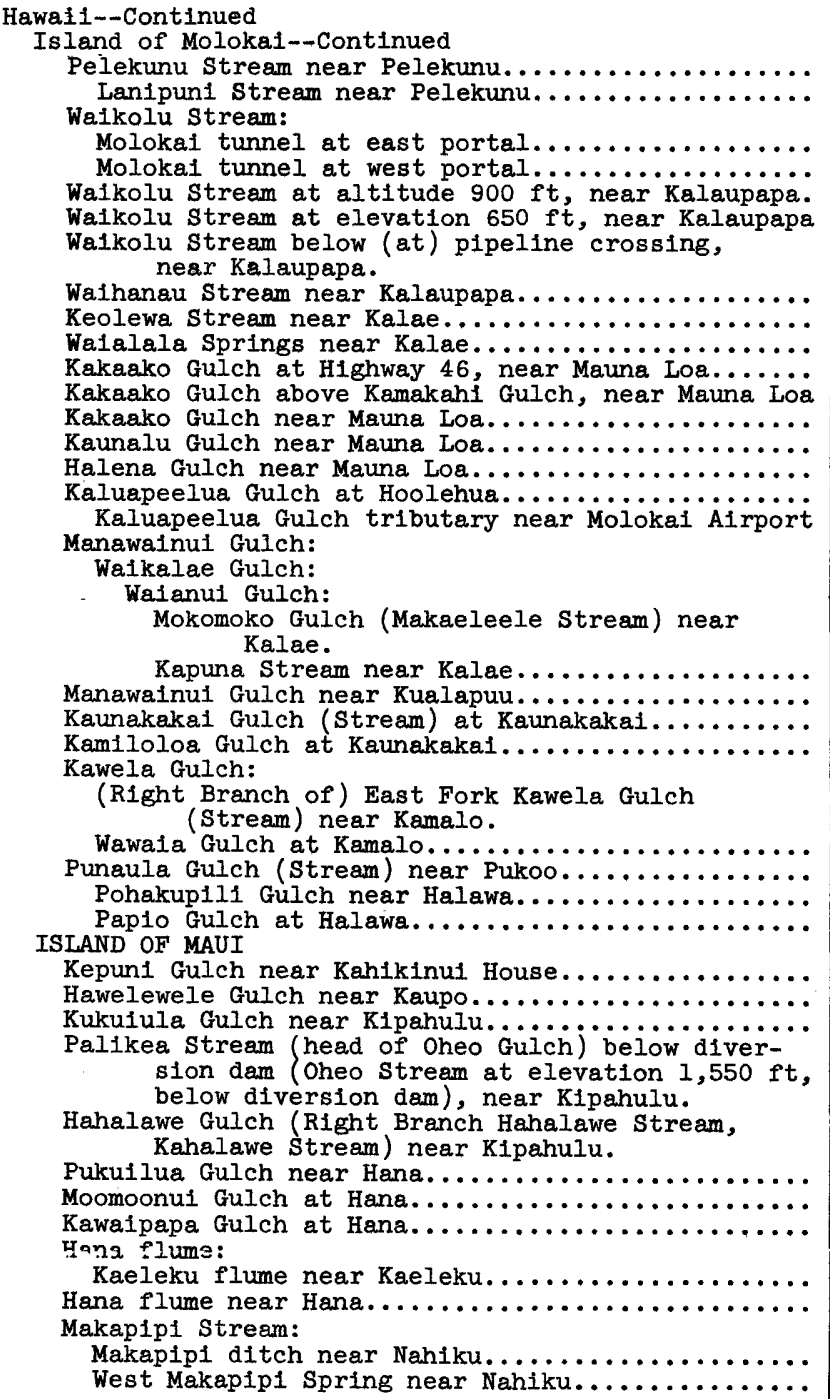 & $\begin{array}{l}16- \\
4040 \\
4050 \\
4051 \\
4053 \\
4055 \\
4060 \\
4080 \\
4090 \\
4100 \\
4110 \\
4113 \\
4113.2 \\
4114 \\
4116 \\
4116.4 \\
4118 \\
4119 \\
\\
4120 \\
4130 \\
4135 \\
4140 \\
4141 \\
4150 \\
4154 \\
4160 \\
4190 \\
4195 \\
5001 \\
5003 \\
5008 \\
5010 \\
\\
5020 \\
\\
5024 \\
5028 \\
5029 \\
5030 \\
5040 \\
5060 \\
5065 \\
\end{array}$ & $\begin{array}{l}2.62 \\
1.09 \\
- \\
1.99 \\
2.99 \\
3.68 \\
1.18 \\
.18 \\
- \\
.55 \\
1.40 \\
5.34 \\
.28 \\
2.07 \\
1.46 \\
1.56 \\
\\
.23 \\
10.4 \\
6.57 \\
3.24 \\
.45 \\
2.12 \\
.24 \\
.48 \\
.94 \\
1.91 \\
11.3 \\
.76 \\
6.29 \\
.43 \\
.48 \\
.90 \\
5.83 \\
- \\
- \\
- \\
-\end{array}$ & 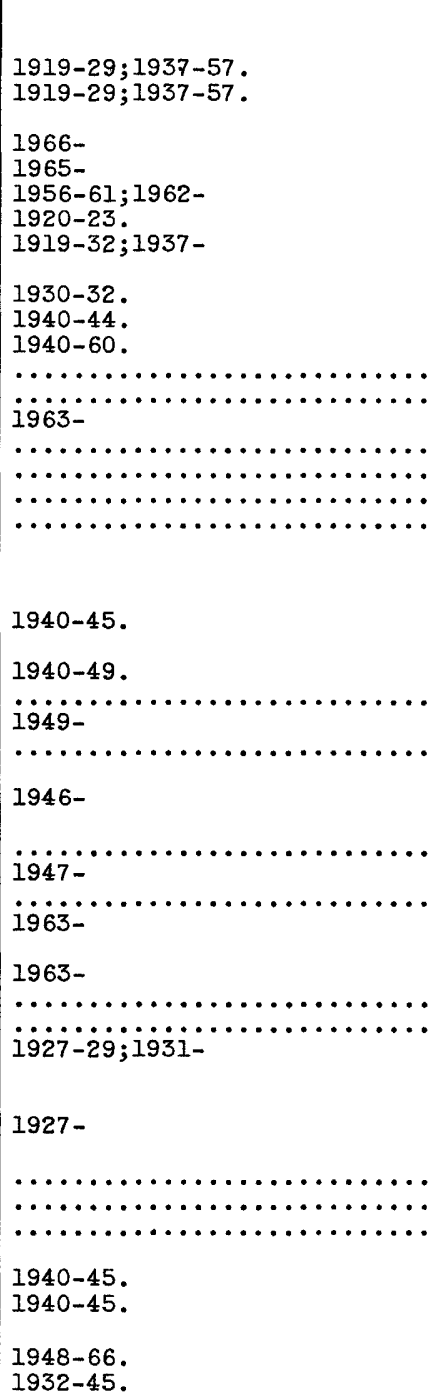 & $\begin{array}{l}1965- \\
1965- \\
1964- \\
1964- \\
1967- \\
1963-67 .\end{array}$ & \\
\hline
\end{tabular}


Makapipi Stream near Nahiku................. 5070

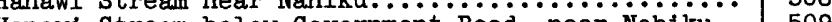

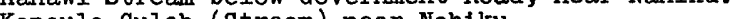

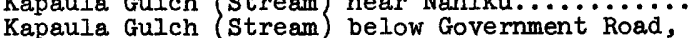

Kapaula Gulch near Nahiku.

Koolau ditch at Nahiku weir, near Nahiku..........

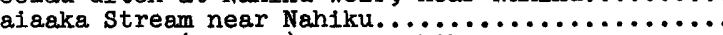

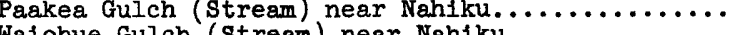

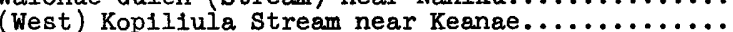

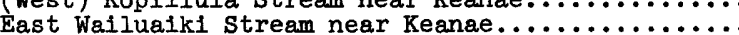

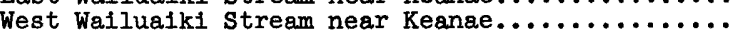

West Wailuenui stream near Keanae...

5200

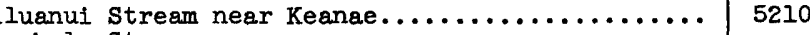

ulu Stream

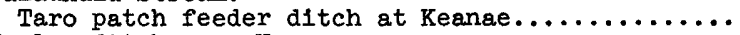

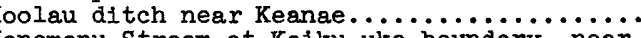

Honomanu Stream at Koiku-uka

Seventh Branch Honomanu Stream at Haiku-uka

boundary, near Kaili1li.

Fourth Branch Honomanu Stream at Kaiku-uka boundary, near Kaililil.

.

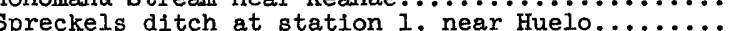
Spreckels ditch at station 2 , near Huelo............

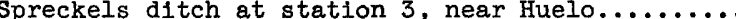
Haipuaena Stream:

Kula diversion from Haipuaena Stream, near Olinda.

Haipuaena Stream at Kula pipeline intake, near Olinda. Haipuaena Stream at Haiku-uka boundary, near
Kaililif.

Third Branch Haipuaena Stream at Haiku-uka boundary, near Kaililli.

First Branch Haipuaena Stream at Haiku-uka

Haipuaena diversion ditch at Kolea Gulch, near Keanae.

Haipuaena Stream above Spreckels ditch, near Huelo (near Huelo).

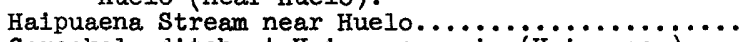

Spreckels ditch at Haipuaena weir (Haipuaena), near Huelo.

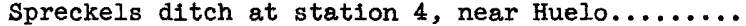
Koolau ditch at Haipuaena, near Huelo............ Manuel Luis ditch

Puohokamoa Stream:

East Branch Puohokamoa stream at Halku-uka boundary, near Kailiili.

Middle Branch Puohokamoa Stream at Haiku-uka

West Branch Puohokamoa Stream at Haiku-uka boundary, near Kallilli.

Puohokamoa Stream above Spreckels ditch, near Huelo (near Huelo).

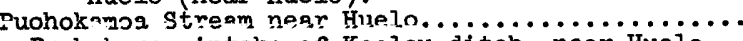

Puohokamoa intake of Koolau ditch, near Huelo... Koolau ditch at Wahinepee, near Huelo $\ldots \ldots \ldots \ldots \ldots$

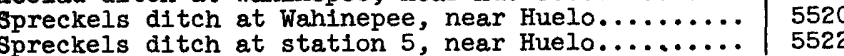

1.93 


\begin{tabular}{|c|c|c|c|c|c|}
\hline \multirow{2}{*}{ Station name } & \multirow{2}{*}{$\begin{array}{r}\text { Station } \\
\text { number }\end{array}$} & \multirow{2}{*}{$\begin{array}{l}\text { Drainage area } \\
\quad(\mathrm{sq} \mathrm{mi})\end{array}$} & \multicolumn{3}{|c|}{ Periods of record } \\
\hline & & & $\begin{array}{l}\text { Daily or monthly figures } \\
\text { (calendar years) }\end{array}$ & $\begin{array}{l}\text { Annual peaks } \\
\text { (f1scal years) }\end{array}$ & $\begin{array}{l}\text { Low-flow measurements } \\
\text { ( } f \text { iscal years) }\end{array}$ \\
\hline 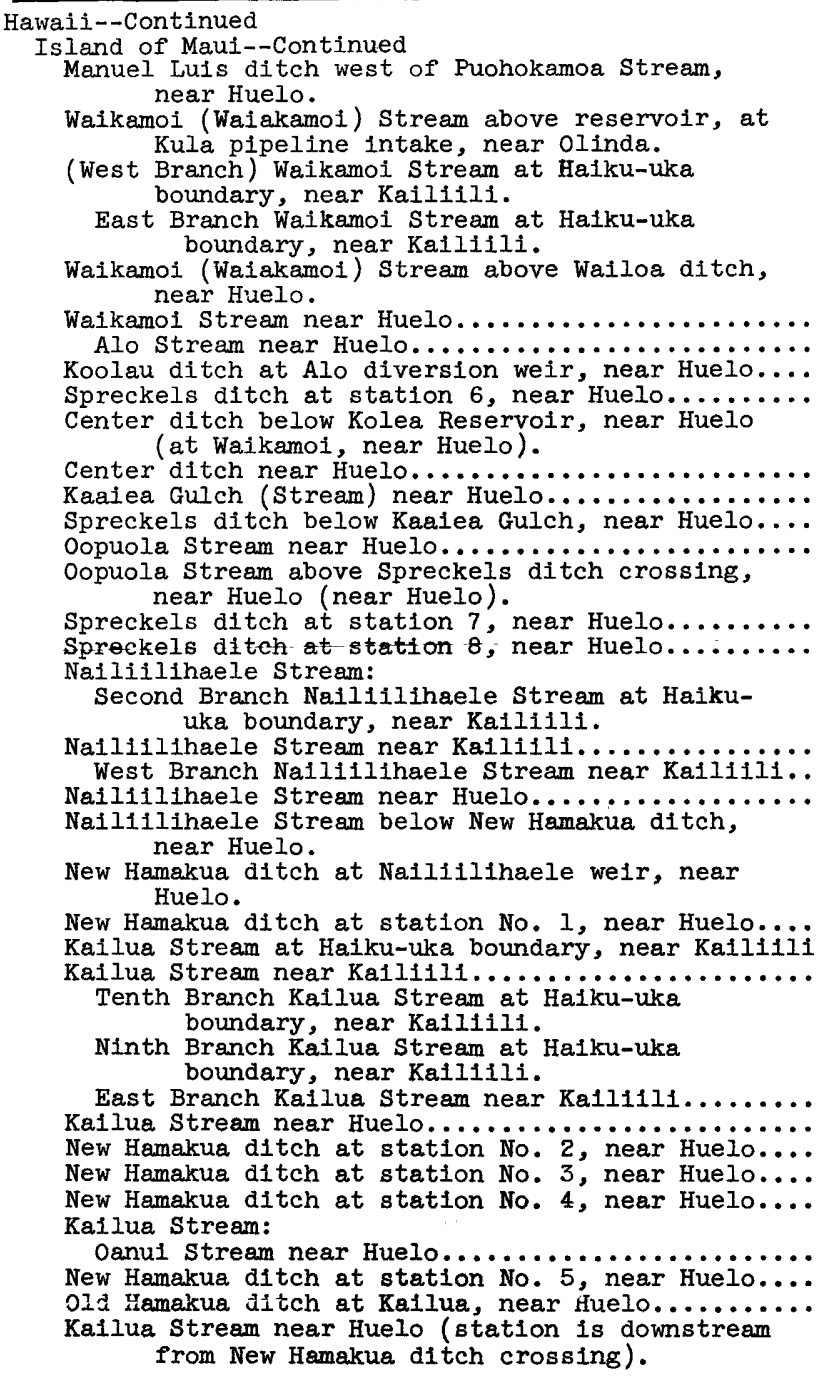 & \begin{tabular}{|l}
$16-$ \\
5525 \\
\\
5528 \\
5540 \\
\\
5545 \\
5550 \\
\\
5560 \\
5570 \\
5580 \\
5600 \\
5610 \\
\\
5620 \\
5650 \\
5655 \\
5660 \\
5670 \\
\\
5675 \\
5680 \\
\\
5690 \\
\\
5691 \\
5697 \\
5700 \\
5710 \\
5720 \\
5730 \\
5740 \\
5745 \\
5750 \\
\\
5760 \\
\\
5762 \\
5770 \\
5780 \\
5790 \\
5795 \\
5800 \\
5820 \\
5830 \\
5840
\end{tabular} & 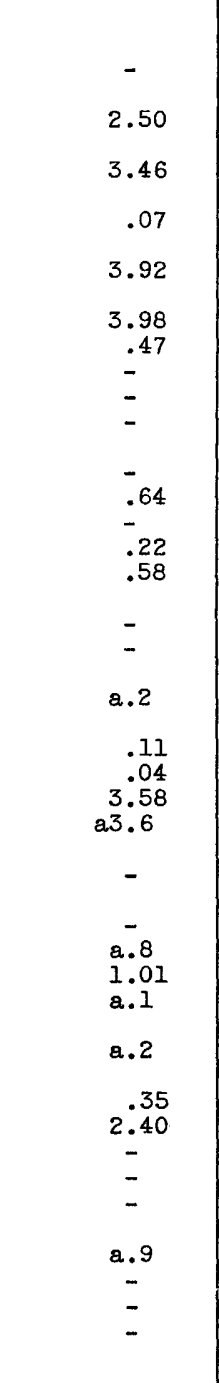 & 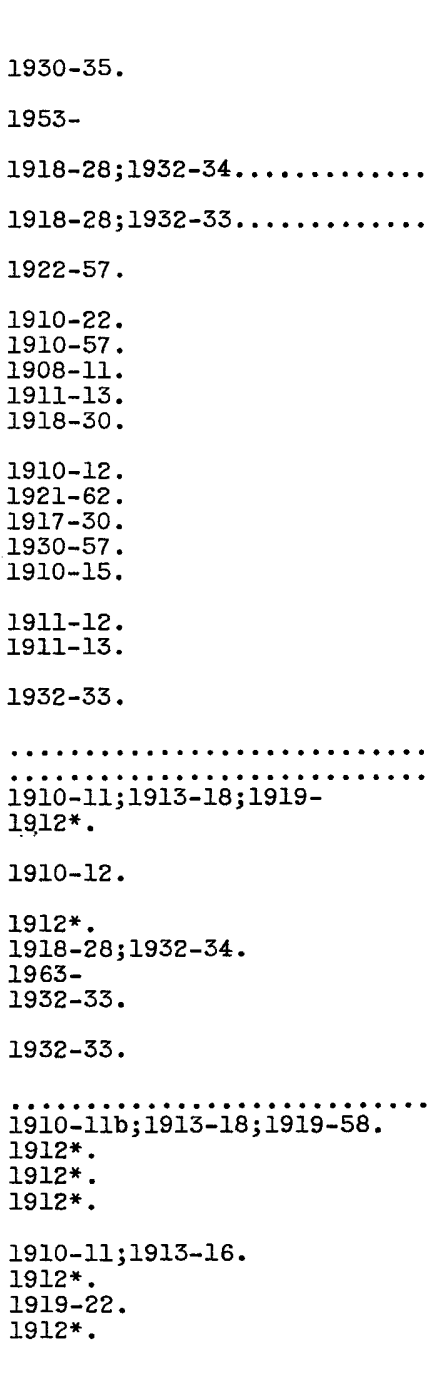 & $\begin{array}{l}\cdots \cdots \cdots \cdots \cdots \\
\cdots \ldots \ldots \ldots \ldots \ldots\end{array}$ & $\begin{array}{l}1963- \\
1963-\end{array}$ \\
\hline
\end{tabular}


Hoolawanui Stream near Huelo.. Wailoa ditch at Honopou, near Huelo.

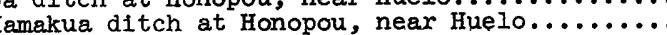

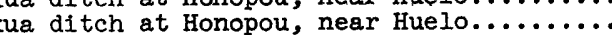
tch siphon, near Huelo. Lowrie ditch to near Huelo.

Honopou Stream above Haiku ditch, near Huelo...... Haiku ditch at Honopou Gulch (at Peahi weir, at Manawai Gulch, at Kapalalaea Gulch), near Kailua.

Honopou Stream below Halku ditch, near Huelo...... New Hamakua ditch at Halehaku weir, near Huelo.... Halehaku Gulch near Kaili1il......................... Huelo.

Halehaku Guich (East Branch Opana Stream) near Huelo.

East Branch Opana Gulch (Stream) at Haiku-uka boundary, near Kaililil.

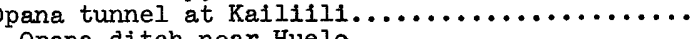

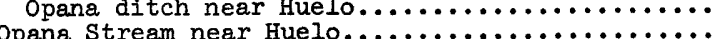
Kauhikoa ditch (old Hamakua ditch) at opana weir, near Huelo.

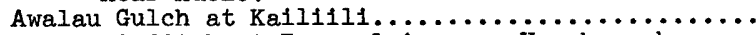

Kaluanui ditch at Puuomalei, near Hamakuapoko......

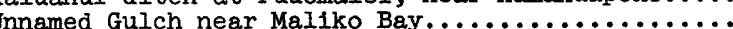
Kalianui Gulch:

Kallanui Gulch tributery near Pukalant.

Kaluapulani Gulch:

Kaluapulant Gulch:

Kaluapulan1 Gulch tributary near Pukalani.....

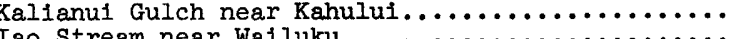

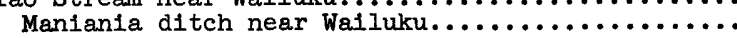

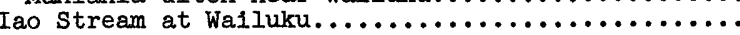

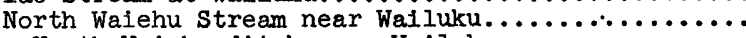
North Waiehu ditch near Wailuku

North Waiehu Stream below North Waiehu ditch, near Wailuku (near Wailuku).

South Walehu Stream near Walluku.

Waihee River (Stream) near Waihee.

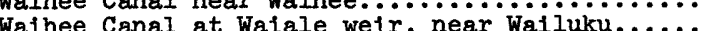

Wathee River (Stream) at dam, near Waihee.

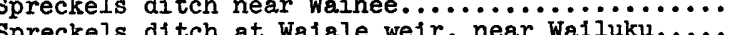

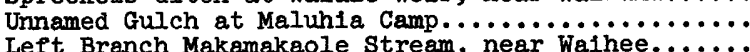
Kahakul stream at altitude I,380 ft, near Honokohau (near Honokohau).

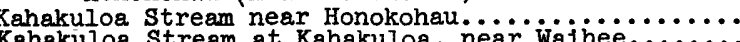
Owaluhi Gulch near Kahakuloa......... Poelua Gulch near Kahakuloa.

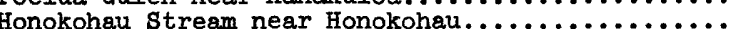
Honokohau ditch at intake, near Honokohau.

Honokohau ditch above Honolua Stream, near Honokohau.

Honolua Stream near Honokohau................. 6230 Honokeana Gulch near Honokahua................ 6234

\begin{tabular}{|c|c|c|}
\hline $\begin{array}{c}1.34 \\
.55 \\
.64 \\
= \\
= \\
2.0 \\
-\end{array}$ & $\mid \begin{array}{l}1910- \\
1912-57 . \\
1910- \\
1922- \\
1918- \\
1918-22 ; 1936-65 . \\
1932-47 ; \\
1910-27 ; 1930-\end{array}$ & \\
\hline a2.2 & $\begin{array}{l}1932-47 \\
1910-28 ; 1930-\end{array}$ & \\
\hline $\begin{array}{l}02.3 \\
-.13 \\
-\end{array}$ & $\begin{array}{l}1932-47 \\
1910-23 \\
1965- \\
1910-12\end{array}$ & \\
\hline 1.44 & $1911 ; 1912^{*}$. & \\
\hline a. 6 & $1932-33$ & \\
\hline $\bar{z}$ & $\begin{array}{l}1965- \\
1910-12 \\
1910-12^{*} \\
1910-28\end{array}$ & \\
\hline $\begin{array}{l}.23 \\
.43\end{array}$ & $\begin{array}{l}1966- \\
1910-12 . \\
\ldots \ldots \ldots \ldots \ldots \ldots \ldots \ldots \ldots \ldots \ldots\end{array}$ & $1963-$ \\
\hline 1.17 & & $1967-$ \\
\hline $\begin{array}{l}.45 \\
17.9 \\
26.0 \\
-\end{array}$ & $\begin{array}{l}\ldots \ldots \ldots \ldots \ldots \ldots \ldots \ldots \ldots \ldots \ldots \\
\text { igio-is. } \\
1910-13 .\end{array}$ & $\begin{array}{l}1963- \\
1967-\end{array}$ \\
\hline $\begin{array}{l}8.24 \\
.90 \\
- \\
0.9\end{array}$ & $\begin{array}{l}1910-10 . \\
1912-15 \ldots \ldots \ldots \ldots \ldots \ldots \ldots \\
1910-11 ; 1916-17 . \\
1911 .\end{array}$ & $1951-$ \\
\hline $\begin{array}{c}a .7 \\
- \\
a 3.9 \\
- \\
- \\
a 3.9 \\
-\end{array}$ & $\begin{array}{l}1910-11 ; 1913-17 . \\
1913 . \\
1913-17 . \\
1910-11 ; 1912 * . \\
1911-12 . \\
1910-12 . \\
1910-13 . \\
1910-11 .\end{array}$ & \\
\hline $\begin{array}{l}.12 \\
a .4 \\
a 1.5\end{array}$ & $\begin{array}{l}\text { i939-52 } \\
1913 ; 1914 .\end{array}$ & $1963-$ \\
\hline $\begin{array}{l}3.47 \\
24.0\end{array}$ & $\begin{array}{l}1939-43 ; 1947- \\
1912-13\end{array}$ & \\
\hline $\begin{array}{l}.20 \\
1.18 \\
4.11 \\
- \\
-\end{array}$ & 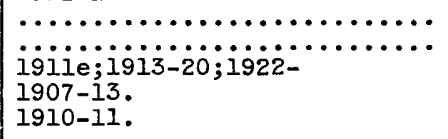 & $\begin{array}{l}1965- \\
1965-\end{array}$ \\
\hline $\begin{array}{l}a 2.9 \\
.59\end{array}$ & $\mid 1913-17$ & $1963-$ \\
\hline
\end{tabular}




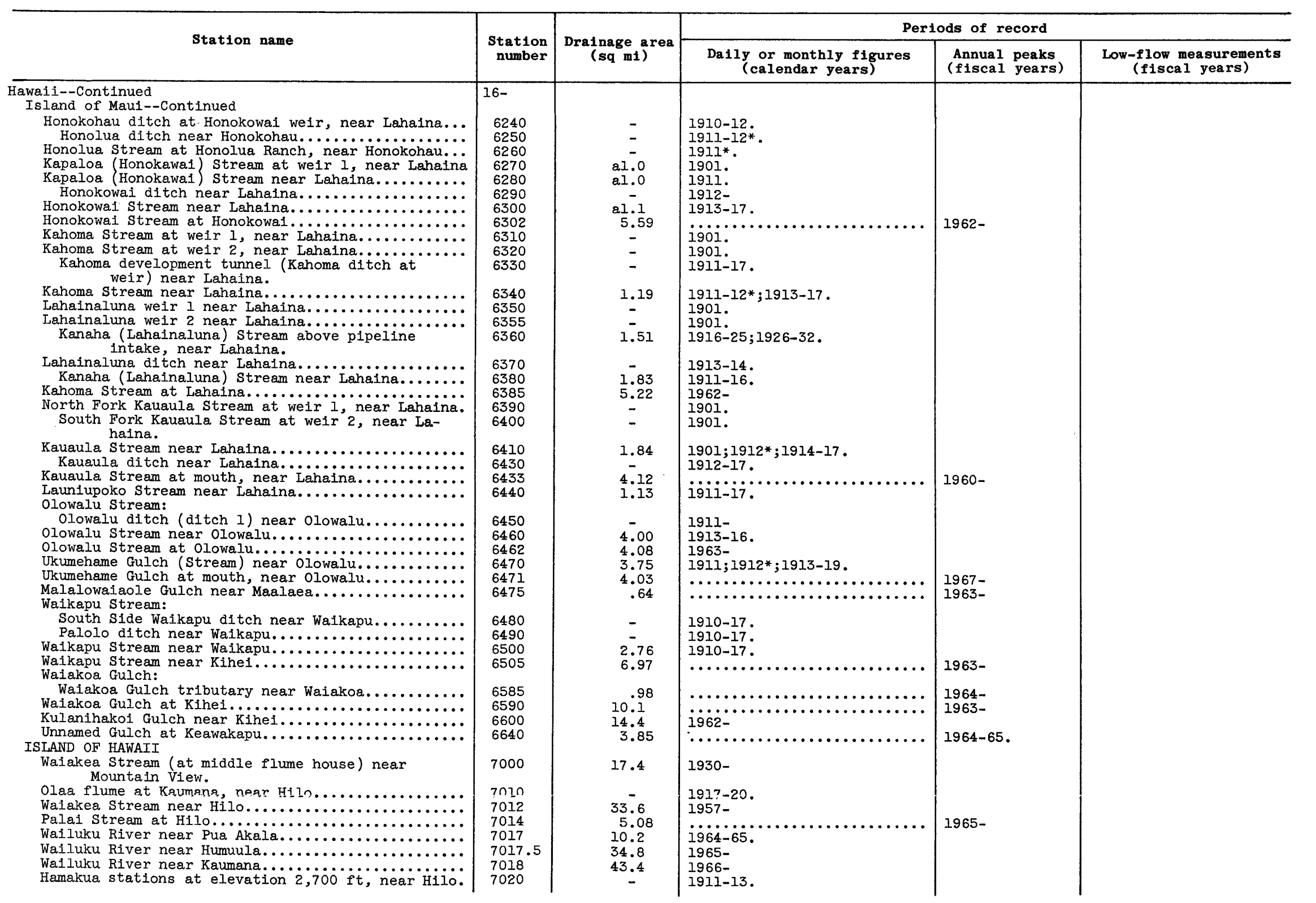


Walluku River at Pukamaui, near Hilo............ ing (above Hilo Boarding scho

Intake, near Hilo), at Pilhonua. Hilo Boarding School ditch at intake, near

Kapehu Stream:

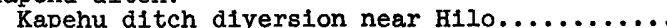
Kapehu ditch near Hilo n...... Kapehu Stream at Pilhonua, near Hilo............

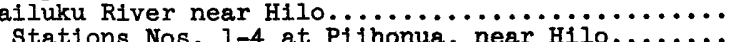

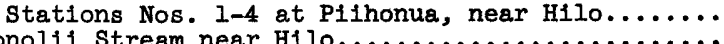
Honoli1 Stream near Hilo $\ldots \ldots \ldots \ldots \ldots \ldots \ldots \ldots \ldots \ldots$ Kalao mauka Stream near Hilo...

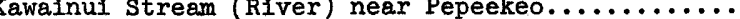

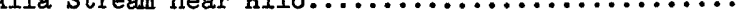
Kapehu Stream near Pepeekeo.................. Pohakupuka Stream near Papaaloa................. Manowa1opae Stream near Laupahoehoe..............

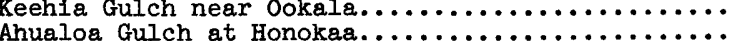
Ahualoa Gulch at Honokaa...................

(East Fork of Left Branch) Honokaia Gulch tribu-
tary near Honokaa.

Kawainul Stream (head of Walloa stream) above tch, near Kamuela.

Upper Hamakua ditch below Kawaiki Stream near Kamuela.

Kawainui Stream at elevation 2,120 ft, near Waipio Branch 3 Kawainui Stream at elevation 1,700 ft,

Kawainui Stream at elevation 1,435 ft, near Waipio Branch 2 Kawainui stream at elevation 1,405 ft, Branch 1 Kawainui Stream at elevation 1,380 ft, near Waipio.

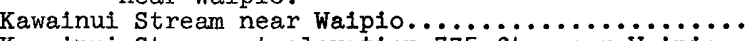
Kawainui Stream at elevation $775 \mathrm{ft}$, near Walpio. Alakahi Stream above Upper Hamakua ditch near

Alakahi Stream at elevation 1,200 ft, near

Alakahi Stream at elevation $730 \mathrm{ft}$, near Waipio. Kolawe Stream at elevation 1,120 ft, near Waipio Koiawe Stream at elevation $610 \mathrm{ft}$, near Waipio..

Waipio River below Kolawe Stream near Waipio...... Waima Stream at elevation $790 \mathrm{ft}$, near Walp1o... Waima Stream at elevation $385 \mathrm{ft}$, near Waipio... Waipio River below Waima stream, near Waipio...... Upper Hamakua (Hamakua) ditch at Puualala and reservoir 3 weirs (at main weir, Puualala),

Lower (New) Hamakua ditch at main weir, near Kukuihaele.

Lower Hamakua ditch wasteway, near Kukuihaele... Lower Hamakua ditch below main weir, near Kukuihaele.

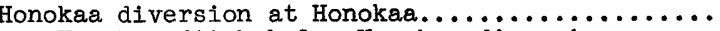
Lower Hamakua ditch balow Honotsa divergion, near Honokaa.

Wailikahi (Waimanuliilii) Stream near Waimanu.... Kaimu Stream near Waimanu...................

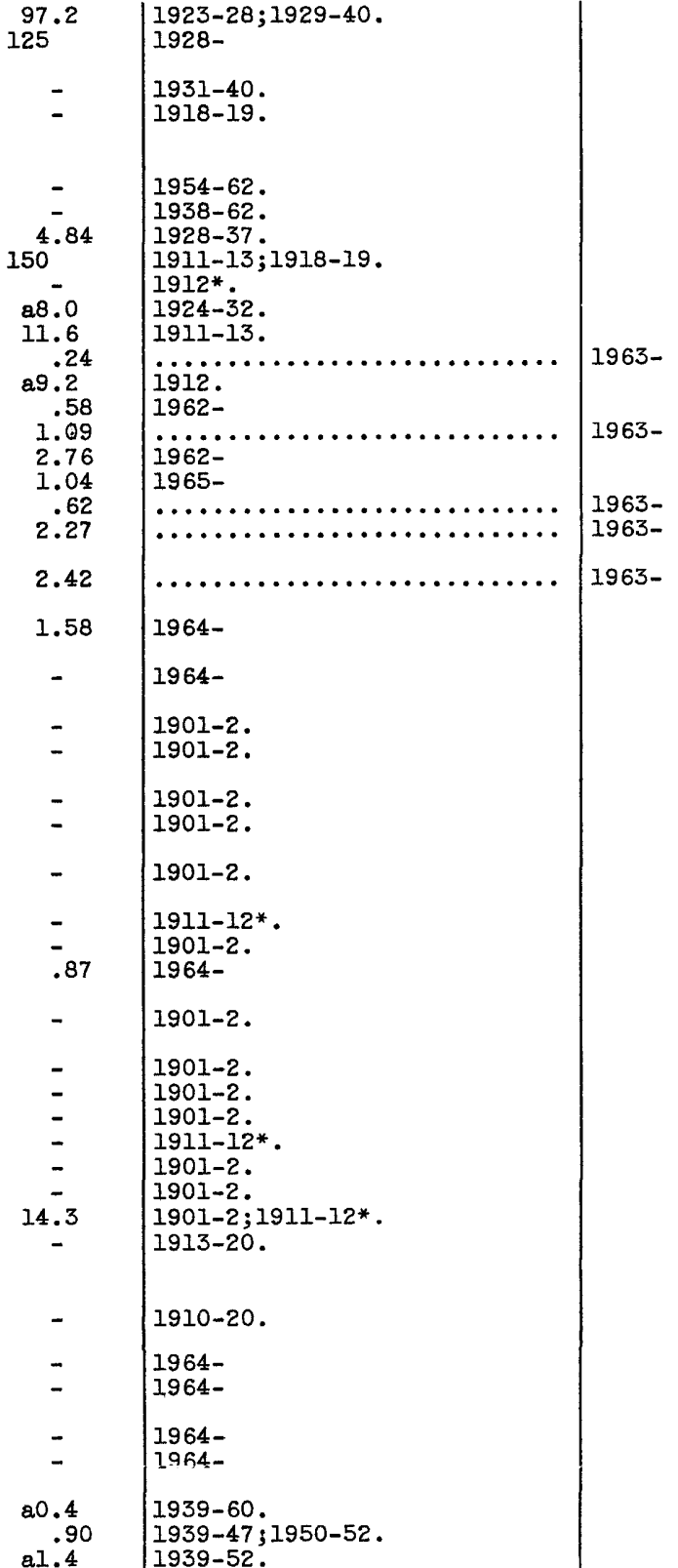




\begin{tabular}{|c|c|c|c|c|c|}
\hline \multirow{2}{*}{ Station name } & \multirow[b]{2}{*}{$\begin{array}{r}\text { Station } \\
\text { number }\end{array}$} & \multirow[b]{2}{*}{$\begin{array}{c}\text { Drainage area } \\
(\mathrm{sq} \mathrm{mt})\end{array}$} & \multicolumn{3}{|c|}{ Periods of record } \\
\hline & & & $\begin{array}{c}\text { Daily or monthly flgures } \\
\text { (calendar years) }\end{array}$ & $\begin{array}{l}\text { Annual peaks } \\
\text { (fiscal years) }\end{array}$ & $\begin{array}{l}\text { Low-flow measurements } \\
\text { ( } \mathrm{f} \text { iscal years) }\end{array}$ \\
\hline 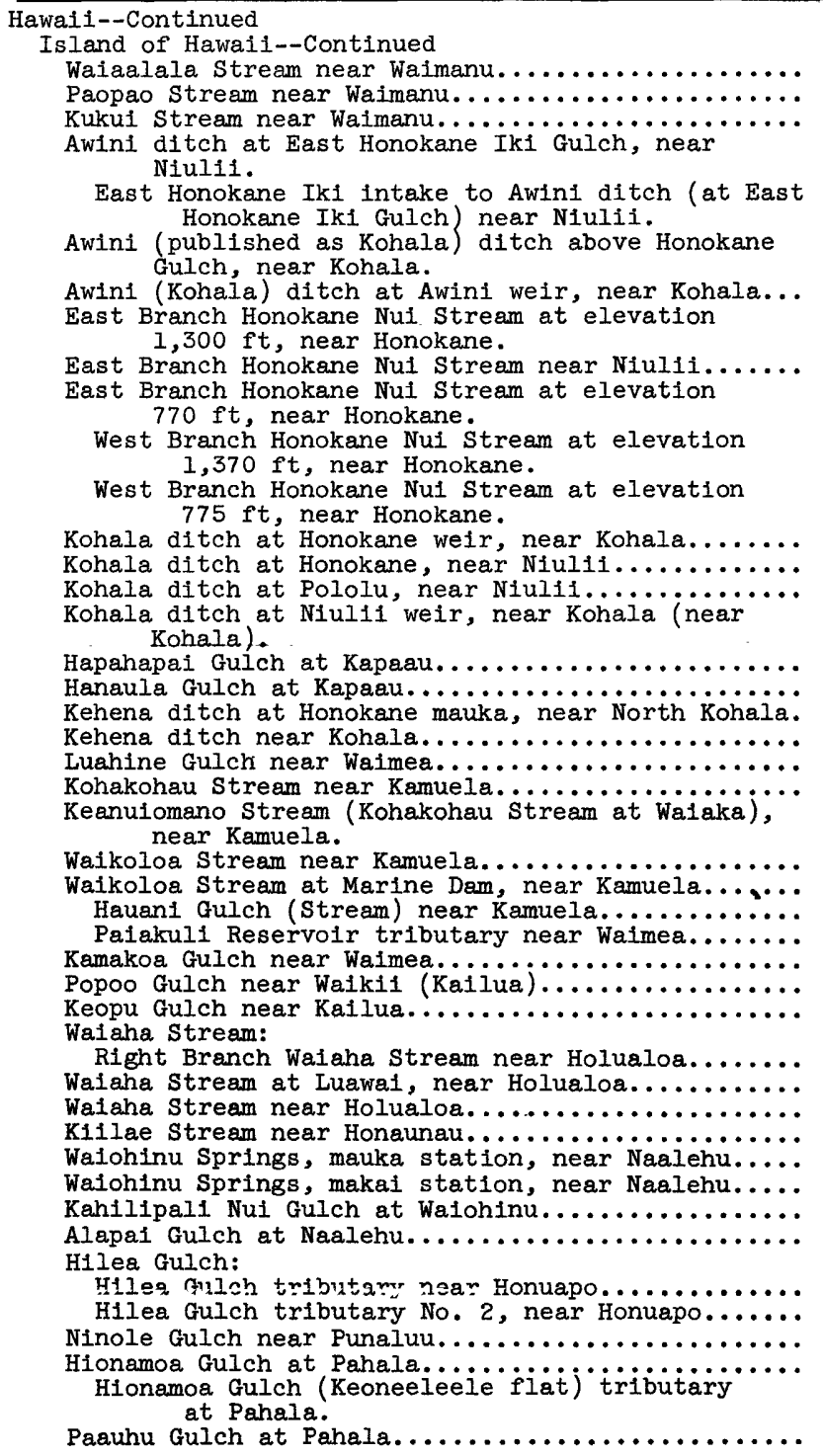 & $\begin{array}{l}16- \\
7400 \\
7410 \\
7420 \\
7430 \\
7440 \\
7450 \\
\\
7455 \\
7470 \\
7475 \\
7480 \\
7490 \\
7495 \\
\\
7500 \\
7509 \\
7510 \\
7520 \\
7526 \\
7528 \\
7540 \\
7550 \\
7558 \\
7560 \\
7565 \\
7570 \\
7580 \\
7590 \\
7590.4 \\
7590.6 \\
7590.8 \\
7591.8 \\
7592 \\
7593 \\
7595 \\
7598 \\
7600 \\
7610 \\
7612 \\
7620 \\
7510 \\
7650 \\
7670 \\
7700 \\
7702 \\
7705 \\
\end{array}$ & $\begin{array}{c}a .2 \\
0.6 \\
- \\
- \\
- \\
- \\
- \\
4.96 \\
- \\
- \\
- \\
- \\
- \\
- \\
- \\
1.52 \\
.21 \\
- \\
.32 \\
2.34 \\
4.30 \\
.78 \\
1.18 \\
.47 \\
.27 \\
23.6 \\
2.61 \\
1.87 \\
8.74 \\
9.35 \\
.67 \\
- \\
.47 \\
2.90 \\
11.7 \\
4.53 \\
14.0 \\
5.87 \\
.42 \\
1.42\end{array}$ & 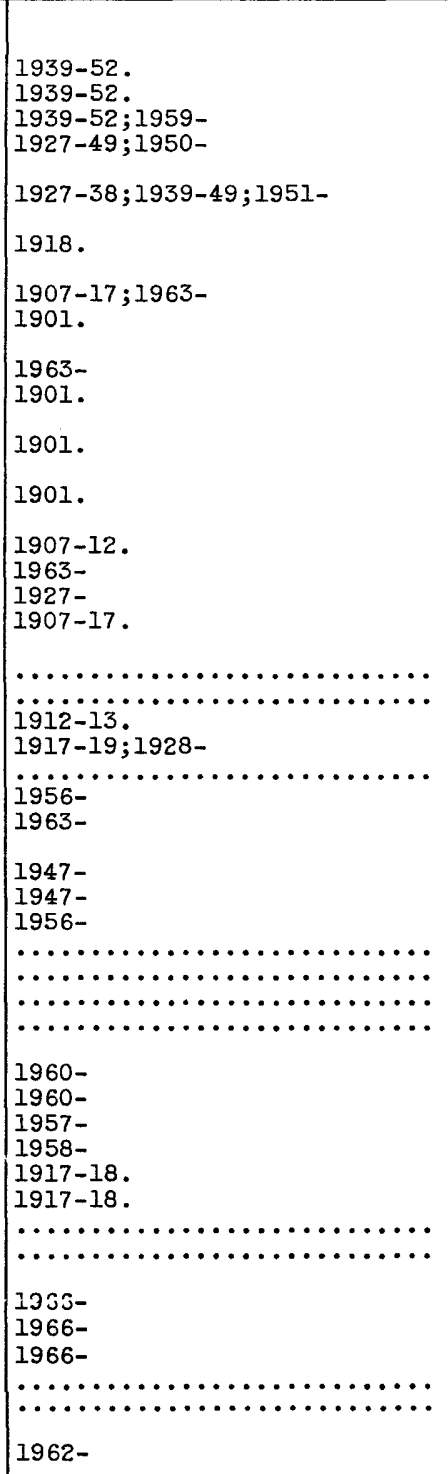 & $\begin{array}{l} \\
1962-65 . \\
1963-\end{array}$ & \\
\hline
\end{tabular}




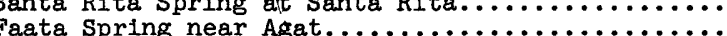

Finile River at Agat.

Taleyfac River near Agat.

Right Branch Cetti River near Umatac.

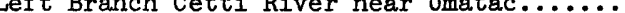

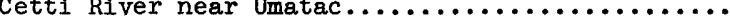

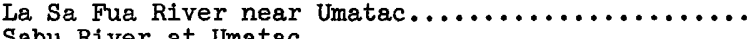

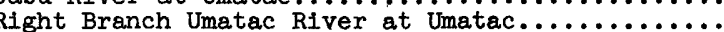

Left Fork of Left Branch Umatac River near Umatac.

Right Fork of Left Branch Umatac River:

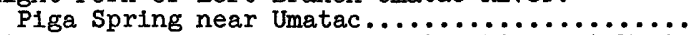

Right Fork of Left Branch Umatac River at Umatac

Umatac River at Umatac.

Geus River above siligin spring tributary, near Merizo.

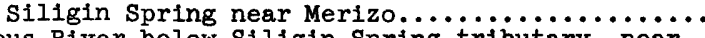

Geus River below Siligin spring tributary, near Merizo.

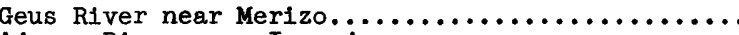

Ajayan River near Inarajan.

(n)

Right Fork of Right Branch Inarajan River near

Inarajan.

Left Fork of Right Branch Inarajan River near Inarajan.

Right Fork of Left Branch Inarajan River near

Inarajan.
Left Fork of Left Branch Inarajan R1ver near Inarajan.

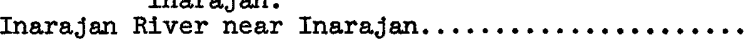

Pauliluc River near Inarajan.

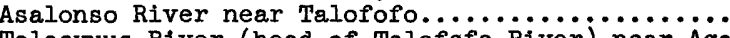

Tolaeyuus River (head of Talofofo River) near Agat

Tolaeyuus River at mouth, near Agat.............................

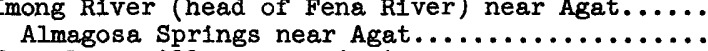

Almagosa Springs near Agat

Fena Dam spillway near Agat.........................

Talofofo River (continuation of Maagas River) near Talofofo.

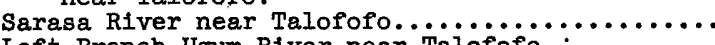

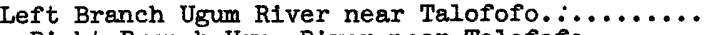

Right Branch Ugum River near Talofofo.........

Ugum River near Talofofo.

Ilg River near Yona.........................................

near Yona.

Lonfit River (head of Pago River) near ordot......

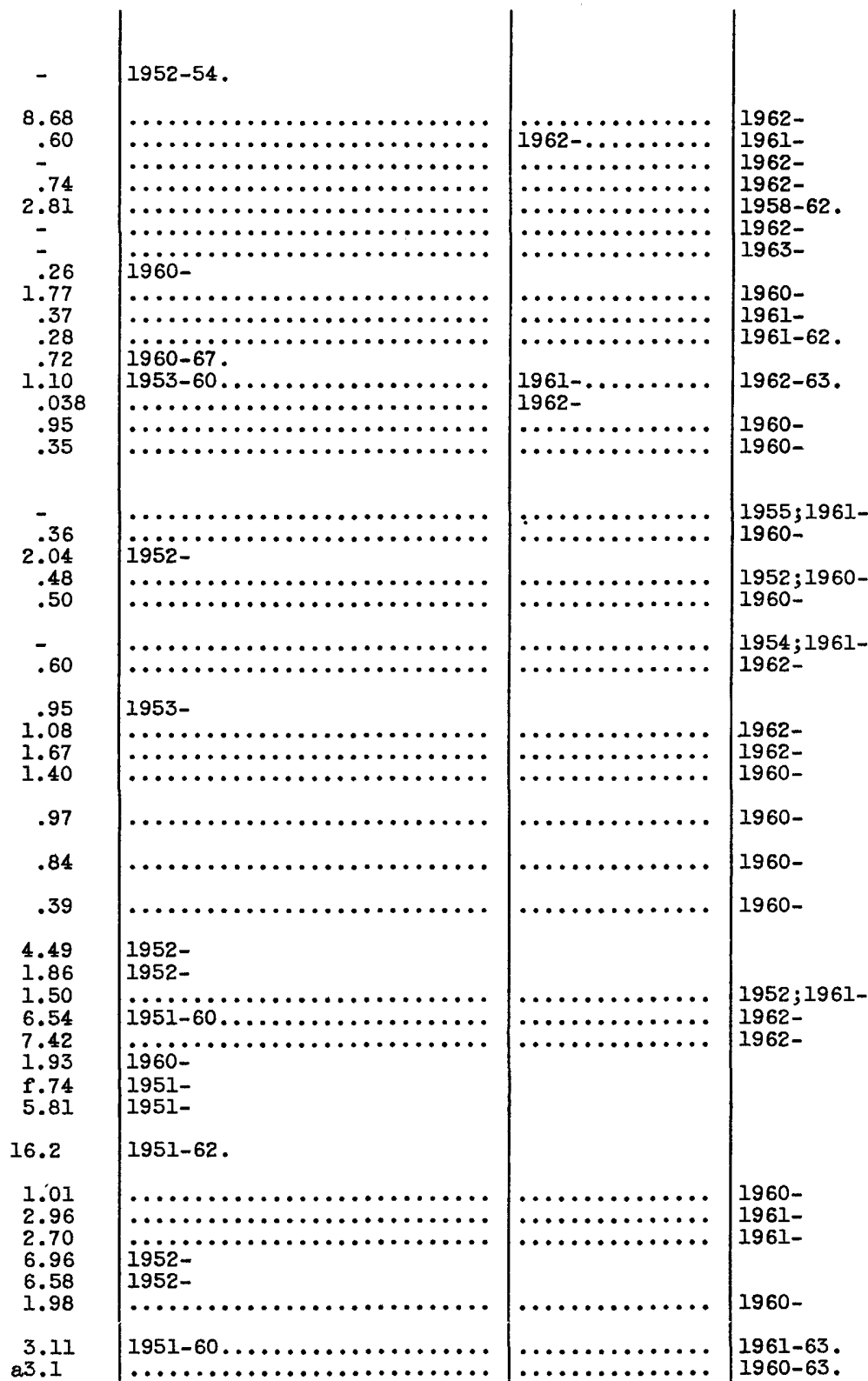




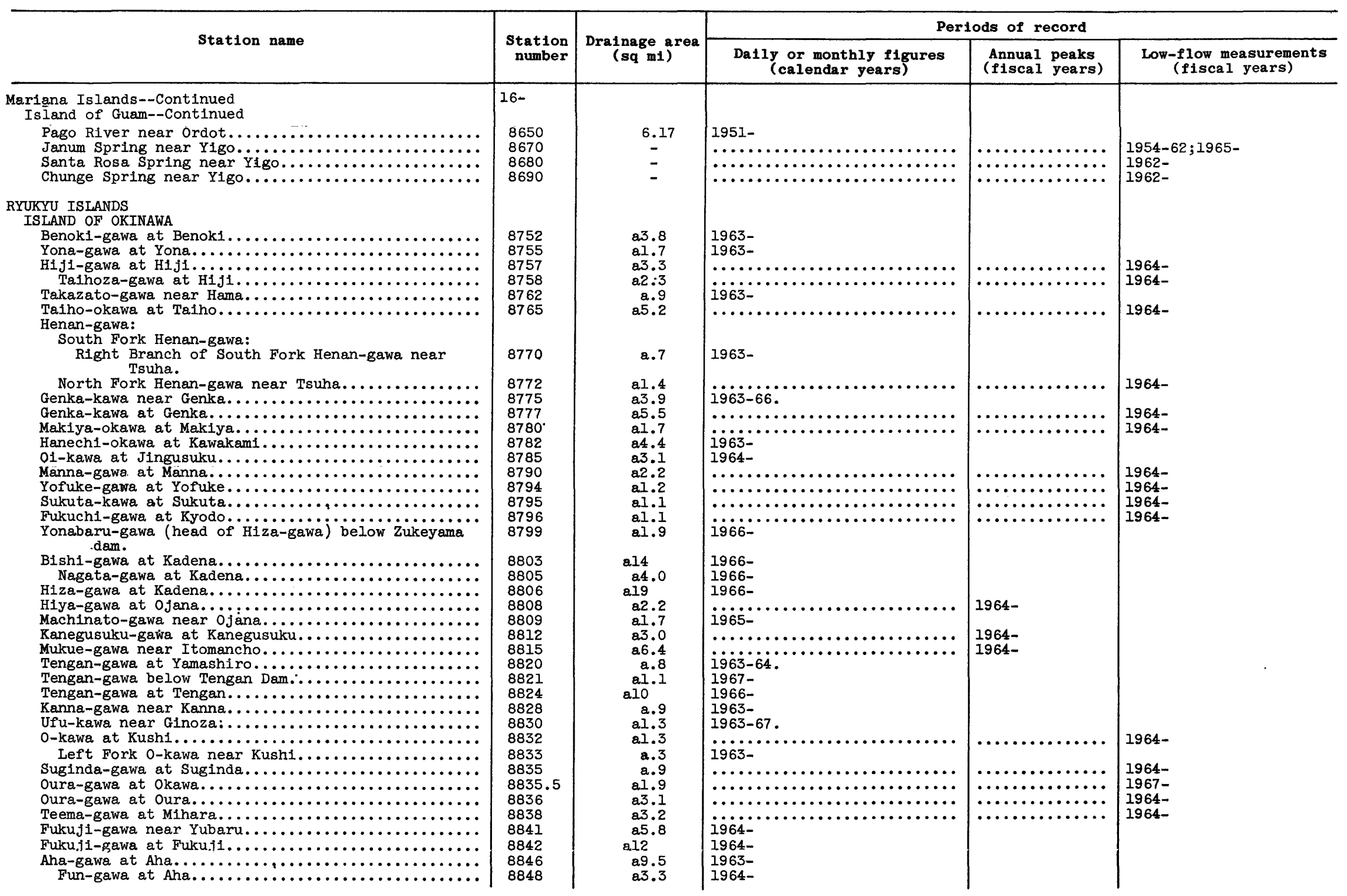



(Village).

Vaisa Stream at Oneona... Vaisa stream at oneona....

Laoulu stream at Aoa (Village) $\ldots \ldots \ldots \ldots \ldots \ldots \ldots \ldots \ldots$

Vaitolu Stream at Aoa.

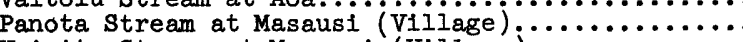

Vaipito Stream at Masausi (Village

Talaloa Stream at Masefau (VIIlage $\ldots \ldots \ldots \ldots \ldots \ldots$

Tagau Stream at Masefau (Village)

Pago Stream (head of Afono Stream):
Pago Stream tributary (Pago Stream) at Afono (Village).

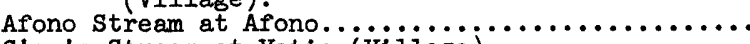

G'ao'a Stream at Vatia (Village)

Leafu Stream at Vatia (Village).

Leua Stream at Fagasa (Village)

Leele Stream at Fagasa (Village).

Lesina Stream at Fagasa (Village)

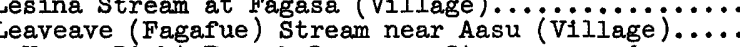

Upper Right Branch Leaveave stream near Aasu....

Lower Right Branch Leaveave Stream near Aasu....

Aasu Stream at Aoloaufou. $\ldots \ldots \ldots \ldots \ldots \ldots \ldots \ldots \ldots \ldots \ldots \ldots$

Aasu Stream near Aa.

Aasu Stream at Aasu.......

Vailolo Stream at Aoloautuai (Aoloau Tuai Village)

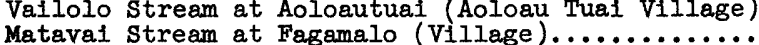

Maloata (Moloata) Stream at Maloata (Moloata

$$
\text { Village). }
$$

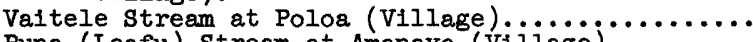

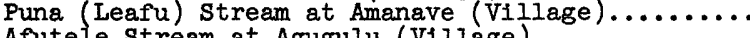

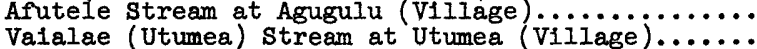

Soonapule (Utuanua Tele) Stream at Seetage (Viliage).

Utanuatele (Utuanua Iti1t1) Stream at Seetage (Village).

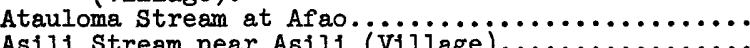

Asil1 Stream near Asil1 (Viliage $\ldots \ldots \ldots \ldots \ldots \ldots$

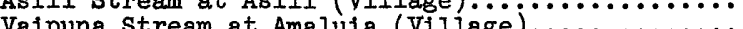




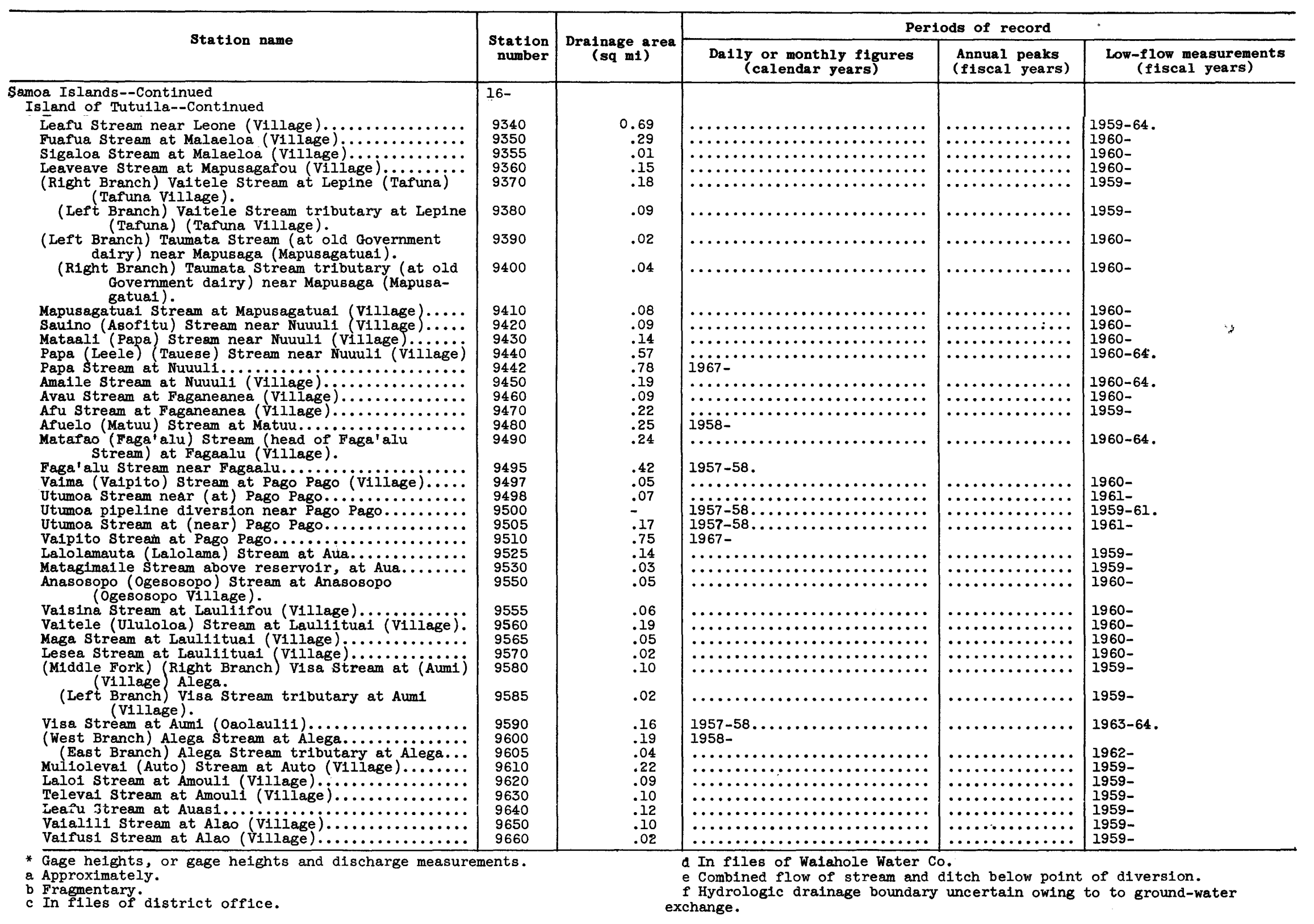


Page

Aahoaka ditch $\ldots \ldots \ldots \ldots \ldots \ldots \ldots \ldots \ldots \ldots$

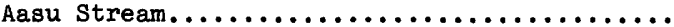

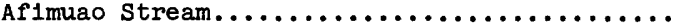
Afono Stream. Afu Stream....

Afuelo Stream. Afutele stream..................... Agana River.

Agfayan River. Ahlo ditch

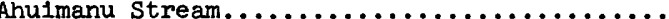

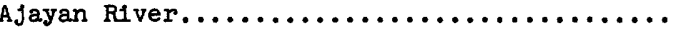

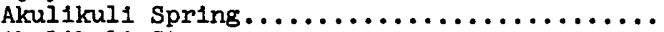

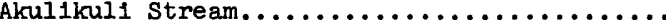

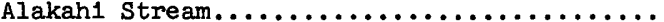
Alapa1 Gulch.

Alega Stream, East Branch.

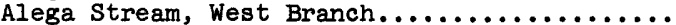

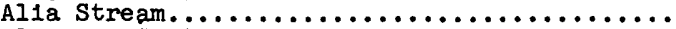
Almagosa Springs. . . . . . . . . . . . . .

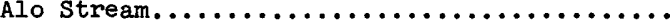

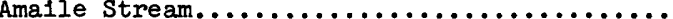

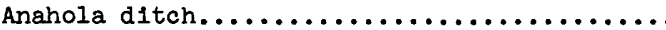
Anahola ditch wasteway

Anahola River.

Anahola Stream. Anahulu River.

Anasosopo Stream.

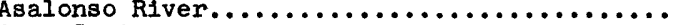
Asan Spring.

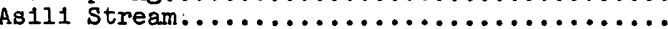

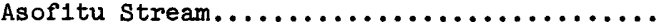

Atantano River

Atauloma Stream.

Auto Stream.

Avau Stream.

Awalau Gulch.

Awint ditch.

Benok1-gawa

B1sh1-gawa.

Center ditch.

Cett1 RIver.

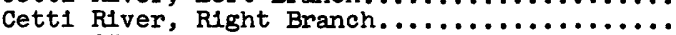

China ditch.

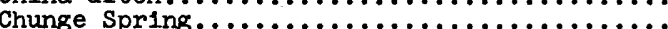

Ditch 1 .

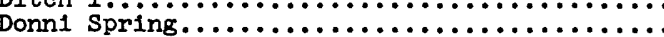

East Branch or Fork. See name of main stream.

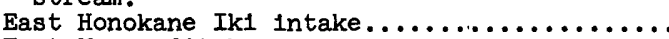

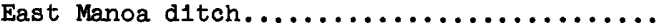

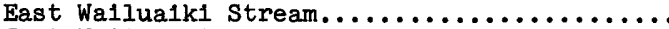

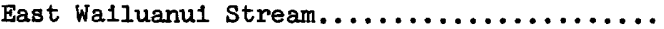

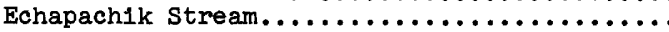

Faata Spring.

Faga'alu Stream.

Fagafue Stream.

(1)

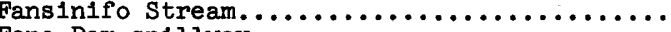
Fena Dam spillway

Finile River.

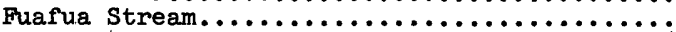

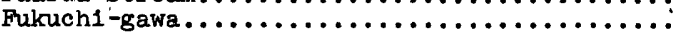

Fukuji-gawa.

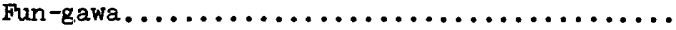

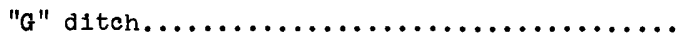

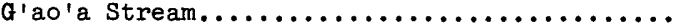

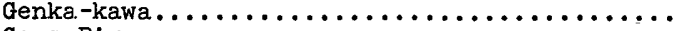

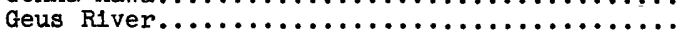

Page

Hahalawe Stream, Right Branch...............

Ha1ku ditch .............................. 13

Ha1ku Stream................................

Halpuaena diversion ditch.................. Il

Ha1puaena stream.......................... II

Halpuaena Stream, First Branch............. 11

Halpuaena Stream, Th1rd Branch.............. 11

Halaulan1 stream......................... 5

Halawa Stream (Isiand of Moloka1)............ 10

Halawa Stream (Island of Oahu) $\ldots \ldots \ldots \ldots \ldots \ldots, 7$

Halehaku Gulch.................................

Halekua Stream............................... 3

Halena Gulch............................. 10

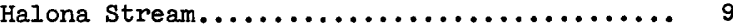

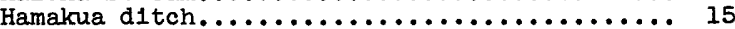

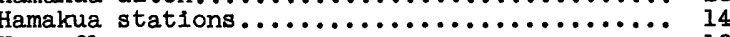

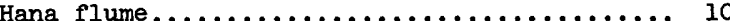

Hanakapiai Stream..................... 5

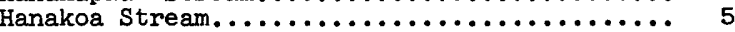

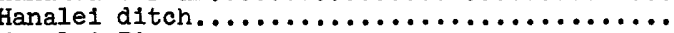

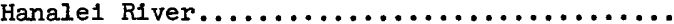

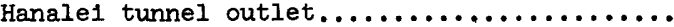

Hanamaulu ditch.......................

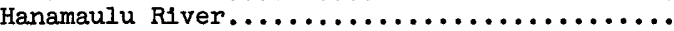

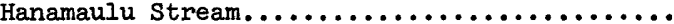

Hanapepe ditch.......................

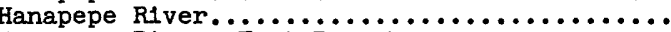

Hanapepe R1ver, East Branch..............

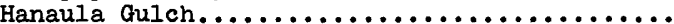

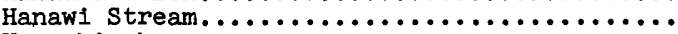

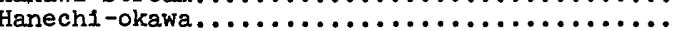

Hapahapa1 Gulch.......................

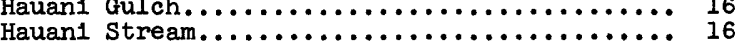

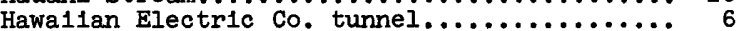

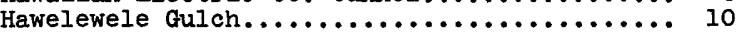

Heela stream.............................. 8

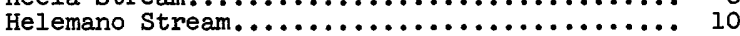

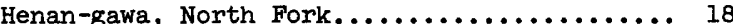

Henan-gawa, Right Branch of South Fork........ 18

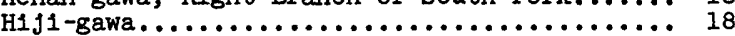

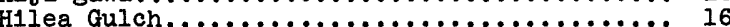

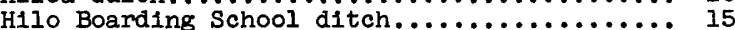

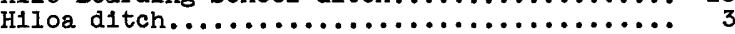

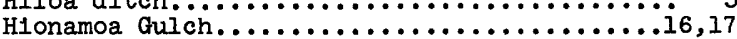

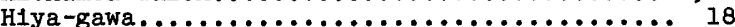

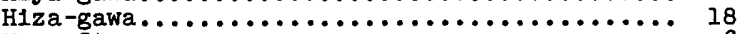

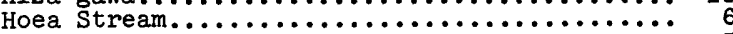

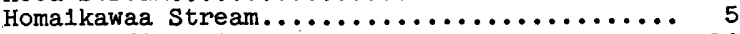

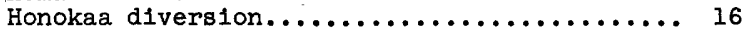

Honokaia Gulch, East Fork of Left Branch...... 15

Honokane Nul Stream, East Branch........... 16

Honokane Nu1 Stream, West Branch............. 16

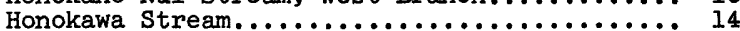

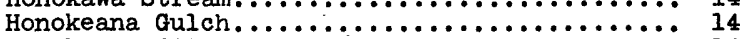

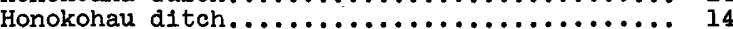

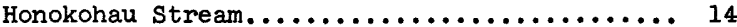

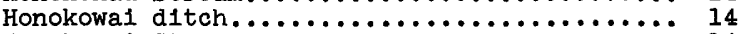

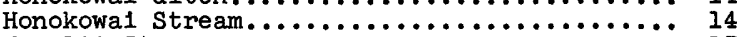

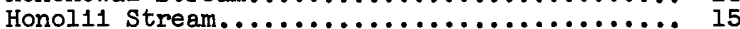

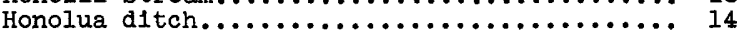

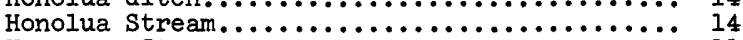

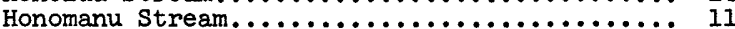

Honomanu Stream, Fourth Branch............. 11

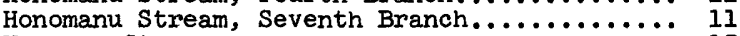

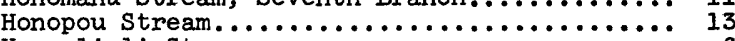

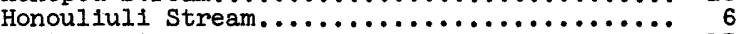

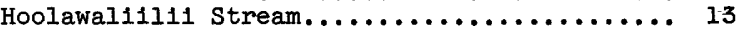

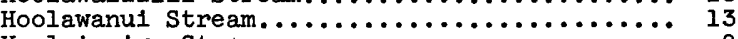

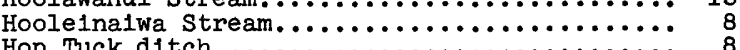

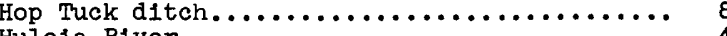

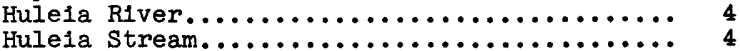

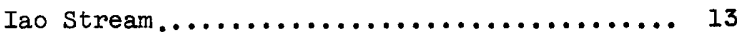

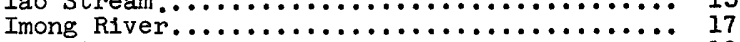

Imor stream $\ldots \ldots \ldots \ldots \ldots \ldots \ldots \ldots \ldots \ldots \ldots \ldots \ldots \ldots \ldots \ldots \ldots \ldots_{19}$ 

Inarajan River, Left Fork of Left Branch..... 17 Inarajan River, Left Fork of Right Branch.... 17 Inarajan RIver, Right Fork of Left Branch..... Inarajan River, Right Fork of Right Branch...

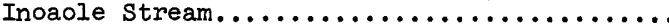

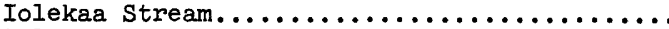

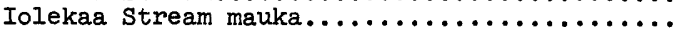

Janum Spring......................

Ka Loko ditch.

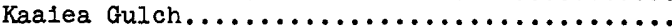

Kaalea Stream.

Kaehulua stream.

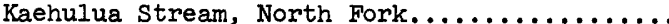

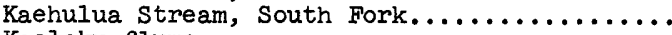

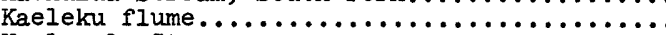

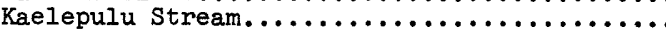

Kahakuloa Stream.....................13, 14

Kahalawe stream, Right Branch............... 10

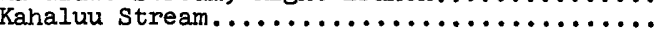

Kahana Stream.

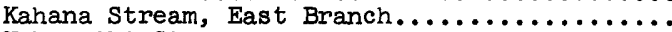
Kahanaiki Stream.

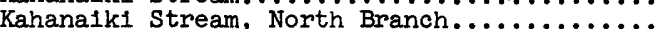

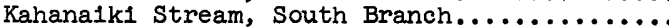

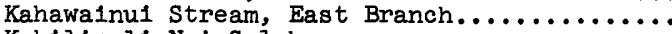

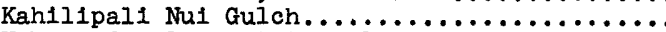

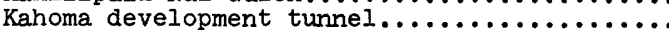
Kahoma ditch.

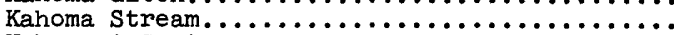
Kahuawa1 Spring

Ka1lua stream Kallua Stream (Island of Oahu $\ldots \ldots \ldots \ldots \ldots \ldots$

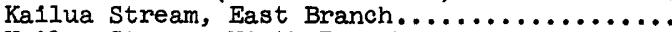

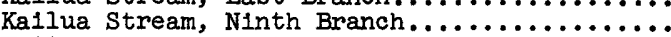

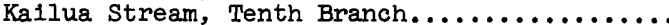

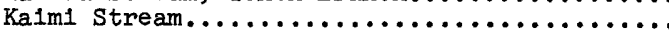
Kaimu Stream.

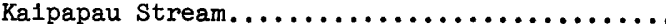

Kakaako Gulch.

Kalalau Stream.

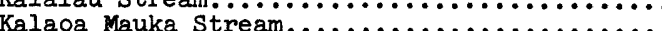

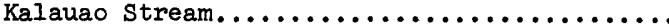

Kallanui Gulch.

Kalihi Stream.

Kaliniwat ditch.

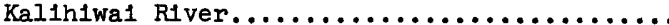

Kalo1 Gulch.

Kaluanu1 ditch.

(1)

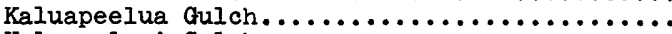

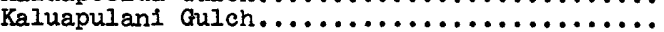

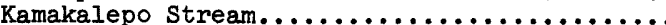

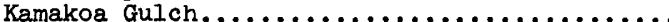

Kamananu1 Stream.

Kamiloloa Gulch.

(n)

Kamooli1 Stream.

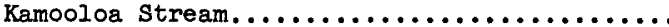

Kanaha ditch.

Kanaha Stream.

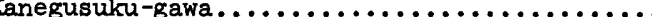

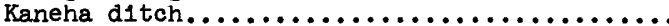

Kaneohe Stream.

Kanna-gawa.

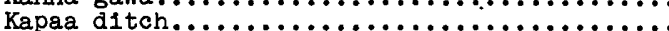

Kapaa River.

Kapaa Stream.

Kapah1 ditch.

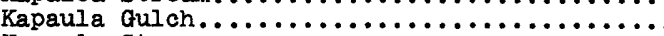

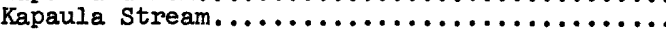

Kapehu ditch.

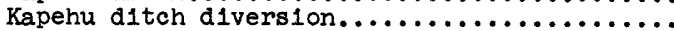

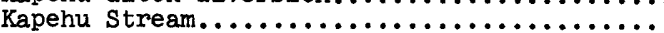

Kapuna Stream.

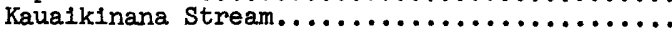

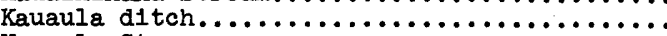

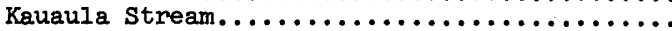

Kauaula Stream, North Fork............. Page

Kauaula Stream, South Fork.............. 14

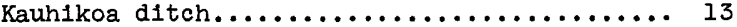

Kaukonahua ditch.......................... 6

Kaukonahua Stream, Left Branch of North

Fork ............................ 6

Kaukonahua stream, North Fork................. 6

Kaukonahua Stream, Right Branch of North

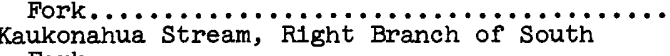

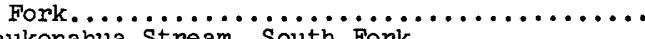

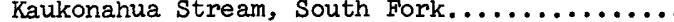

Kaunakakai Gulch..................... 10

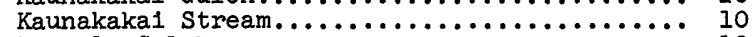

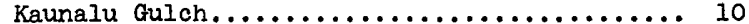

Kaupuni stream..................... 6

Kawa stream....................... 8,9

Kawalkoele stream................... 9

Kawa1ko1 stream..................... 3

Kawa1nu1 Canal...................... 8

Kawa1nu1 R1ver.......................... 15

Kawalnul stream (Isi and of Hawaii).......... 15

Kawalnul Stream (Island of Oaht)............ 9

Kawalnu1 Stream, Branch 1, 2, and $3 \ldots \ldots \ldots \ldots 15$

Kawainu1 Swamp drainage canal............. 8

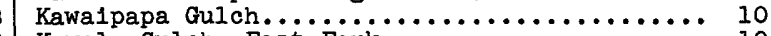

Kawela Gulch, East Fork.................. 10

Kawela Gulch, Right Branch of East Fork...... 10

16 Kawela Stream, East Fork................ 10

14 Kawela Stream, Right Branch of East Fork..... 10

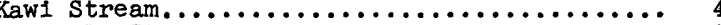

Keaahala stream...................... 8

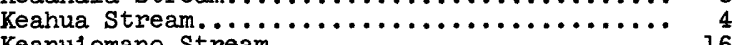

Keanulomano Stream.................... 16

Keeh1a Gulch....................... 15

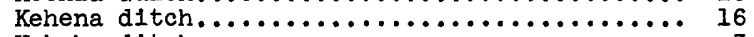

Kekaha ditch.......................... 3

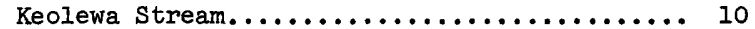

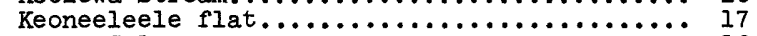

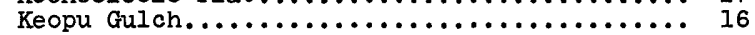

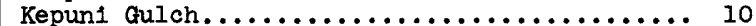

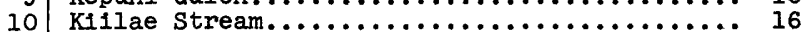

Kipapa stream.............................

Koale stream....................... 3

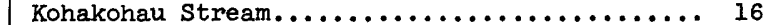

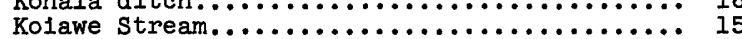

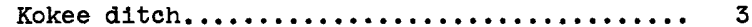

Koloa ditch......................... 4

Koloa Gulch............................ 9

13 Koloa stream....................... 9

Koloa tunnel......................... 4

Koolau ditch (Isiand of Maui)..................

Koolau ditch (Island of Oahu .............. 6

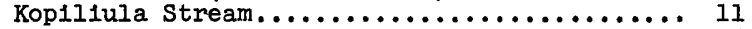

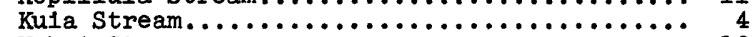

Kukui stream......................... 16

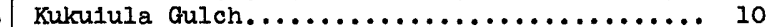

4 Kula diversion......................... 11

4 Kulan1hako1 Gulch $\ldots \ldots \ldots \ldots \ldots \ldots \ldots \ldots \ldots \ldots \ldots \ldots \ldots \ldots \ldots$

14 Kuliouou stream............................ 7

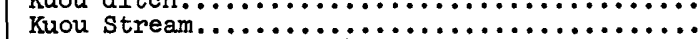

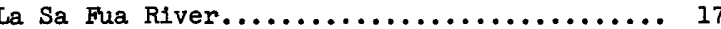

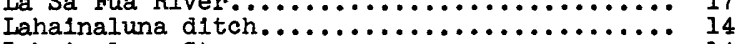

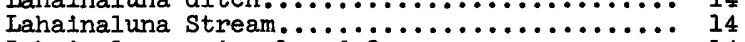
Lahainaluna weirs $i$ and $2 \ldots \ldots \ldots \ldots \ldots \ldots \ldots \ldots . \ldots \ldots \ldots$

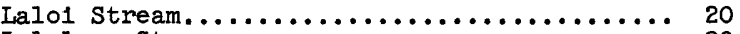

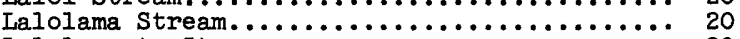

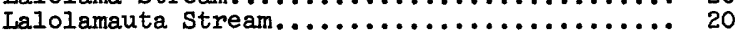

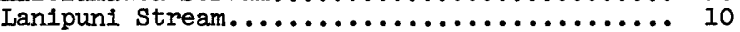

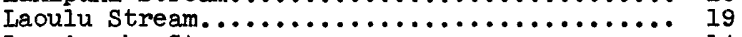

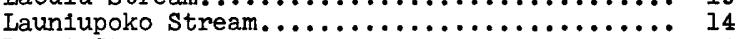

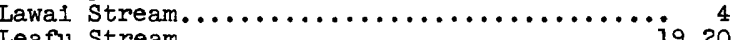

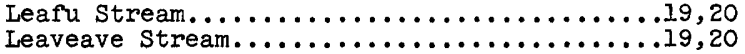

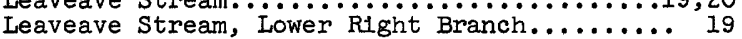


Leaveave Stream, Upper Right Branch....... 19

Lee de Stream................................. Left Branch or Fork. See name of main stream.

Lepa Stream.

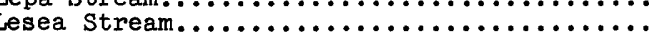

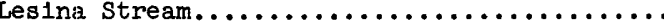

Leua Stream.

Lihue ditch.

Lonf1: River.

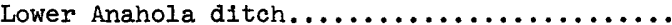

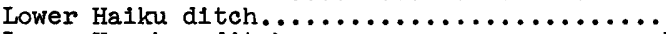

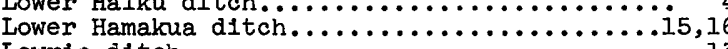

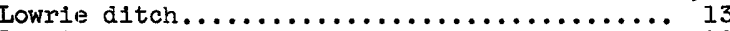

Luahine Gulch..................... 16

Iuakaha we1r.

Luluku Stream.

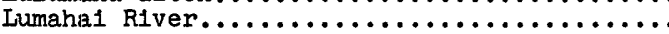

Maagas River.

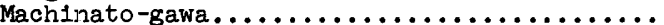

Maga stream.

Ma1111111 Stream.

Main Spring

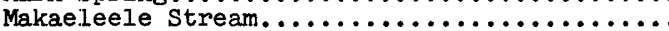

Makaha Stream...

Makaleha ditch.......

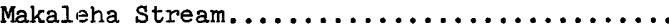

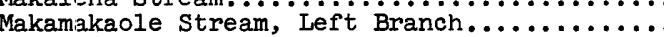

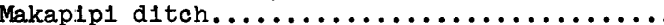

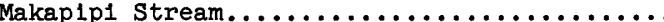

Makaua Stream.

Makawzo ditch.

Makawao flume

Makawao Spring.

Makawao Stream

Makawel1 R1ver

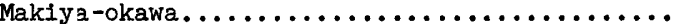

Makua Stream.

Malaekahana Stream

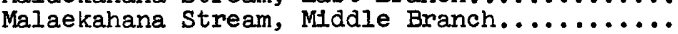

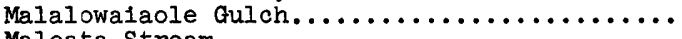

Maloata Stream.

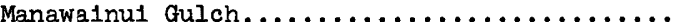

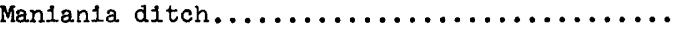

Manoa Stream.

Manoa Stream, East Branch

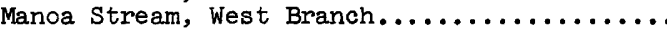

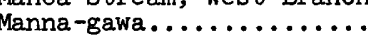

Manowalopae Stream.

Manuah1 Stream.

Manuel Iuf altch.

Mapusagatuai Stream.

an.

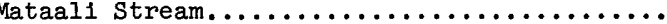

Matafao Stream.

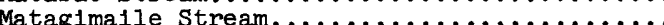

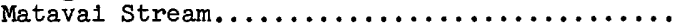

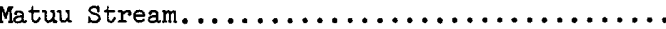

Mauka ditch.

Maunawili ditch

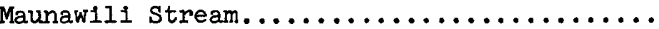

Midale Branch or Fork. See name of main stream.

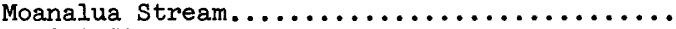

Moh1h1 Stream.

Mokihaha Stream

Mokomoko Gulch.

Moloata Stream

Moloka1 tunnel.

Moole ditch...

Moomoonui

Mukue -gawa

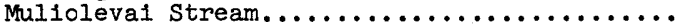

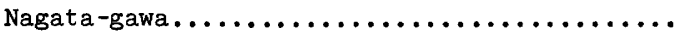

Nahomalu stream..................... 6

Nahomalu valley...................... 6

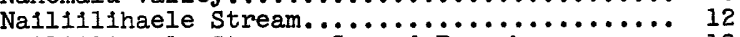

Na1l11lihaele Stream, Second Branch......... 12

Na1l11lihaele Stream, West Branch.......... 12

Nanakul1 Stream..................... 6

20 New Hamakua ditch (İsiand of Hawaij).......... 15

19 New Hamakua ditch (Island of Mau1)..........12,13

19 N1nole Gulch........................ 16

North Branch or Fork. See name of main stream.

North Halawa Stream.................. 7

North Luluku ditch.................... 8

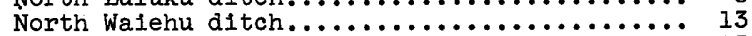

North Waiehu Stream................... 13

North Wallua ditch $\ldots \ldots \ldots \ldots \ldots \ldots \ldots \ldots \ldots \ldots \ldots \ldots \ldots \ldots$
Nuuanu

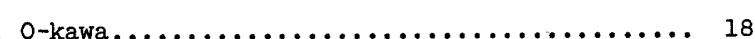

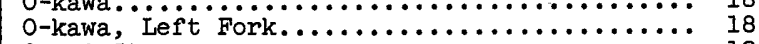

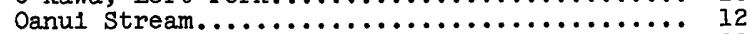

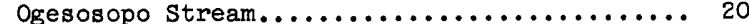

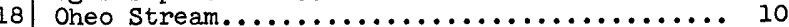

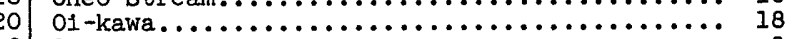

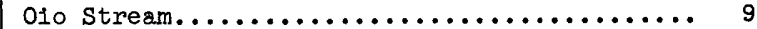

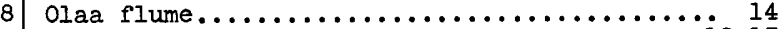

10 Old Hamakua ditch $\ldots \ldots \ldots \ldots \ldots \ldots \ldots \ldots \ldots \ldots \ldots, 12$

olokele ditch..................... 3

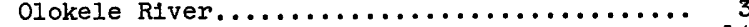

Olowalu ditch...................... 14

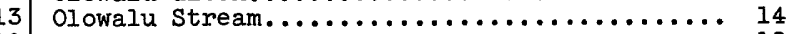

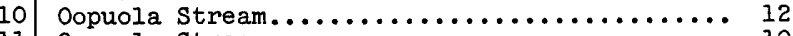

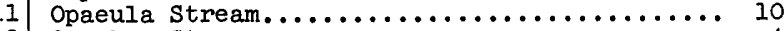

Opaekaa Stream....................... 4

8 Opaekaa Stream, Left Branch............ 4

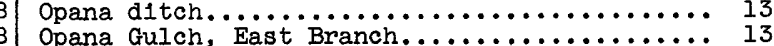

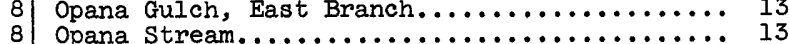

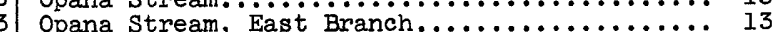

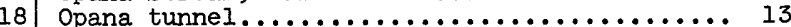

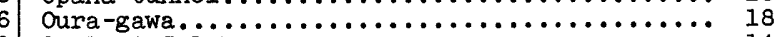

9 Owaluk1 Gulch..................... 14

9 Paakea Gulch...................... 11

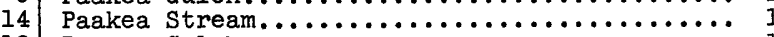

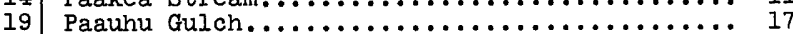

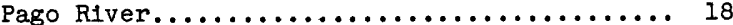

Pago Stream....................... 19

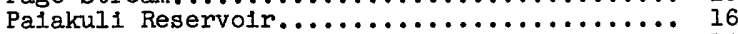

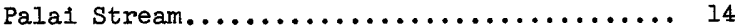

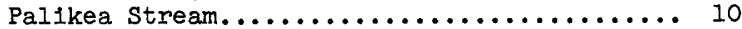

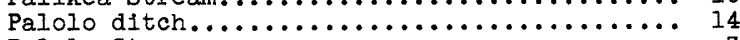

Palolo stream..................... 7

Palolo-Manoa drainage Canal................ ${ }^{7}$

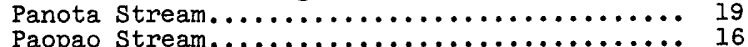

Paopao Stream..................... 16

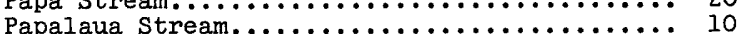

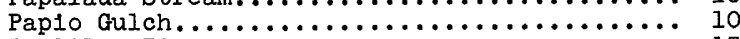

Pauliluc River.................... 17

19 Paumalu Gulch....................... 9

20 Pauoa Stream......................... 7

6 Pearl Harbor Springs................... 6,7

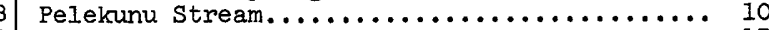

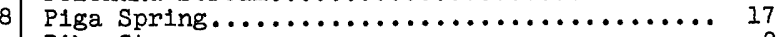

Piho stream P1honua stations Nos. $1-4 \ldots \ldots \ldots \ldots \ldots \ldots \ldots \ldots,{ }^{8}$

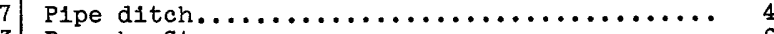

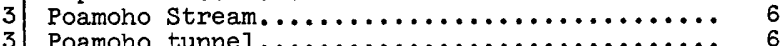

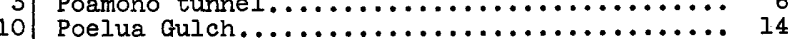

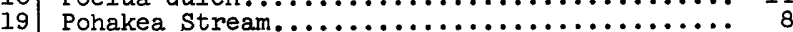

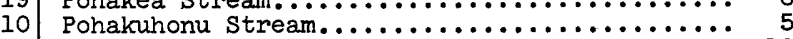

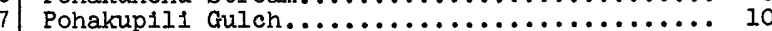

10 Pohakupuka stream................... 15

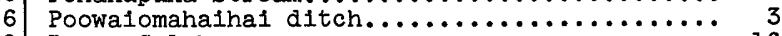

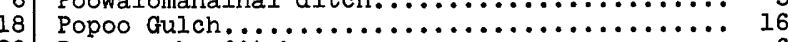

20 Puea makka ditch..................... 6

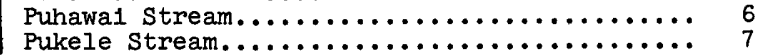


Pukuilua Gulch.

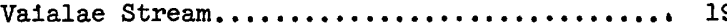

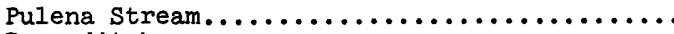
Pump ditch... Valal1l1 stream....................... 20

Puna Stream $\ldots \ldots \ldots \ldots$

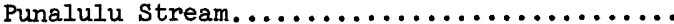

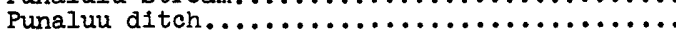
Punaluu Stream.

Punaula Gulch.

Puohokamoa intake.

Puohokamoa Stream.

Puohokamo

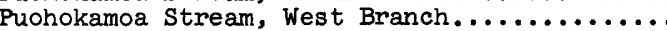

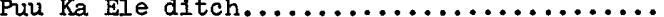

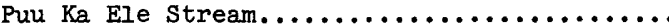

Puukumi Stream.

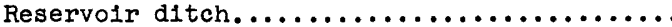

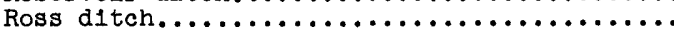

Sabu RIver........................

Santa Rita Spring.

Santa Rosa Spring.

Sarasa RIver $\ldots . .0 .0 .0 .0000000$

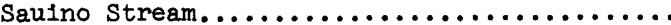

Sigaloa Stream.

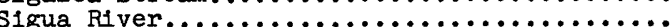

Siligin Spring.

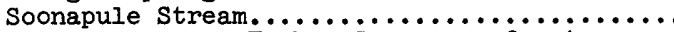

South Branch or Fork. See name of main

stream.

South Walehu ditch................... 13

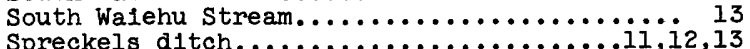

stable storm ditch.....................

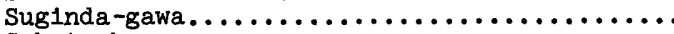

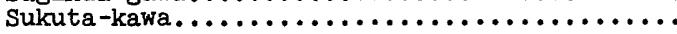

Tagau Stream...................... Taiho-okawa.

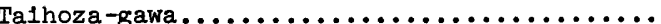

Takazato-gawa.

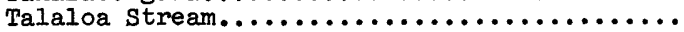

Taleyfac River.

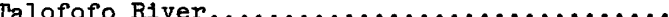

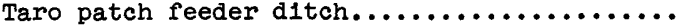

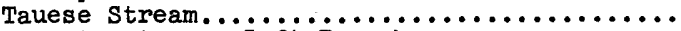

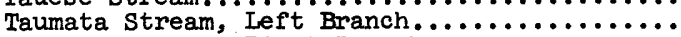

Taumata Stream, RIght Branch.............

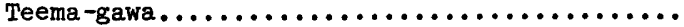

Televal Stream.

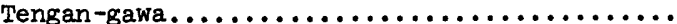

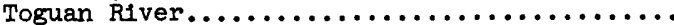

Tolaeyuus River......................

Tunnel ditch......................

Ufu-kawa.......................... Ugum River

Ugum River, Left Branch.................

Ugum Rlver, R1ght Branch..............

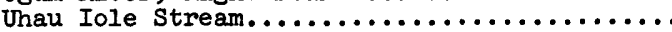

Ukumehame Gulch.....................

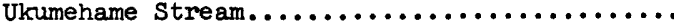

Ulehawa stream.......................

Ululoloa stream.

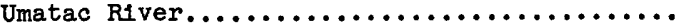

Umatac River, Left Fork of Left Branch.......

Umatac River, Right Branch...............

Umatac River, Right Fork of Ieft Branch......

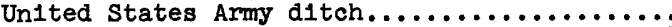

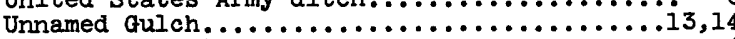

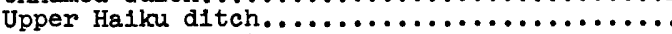

Upper Hamakua ditch....................

Utanuatele Stream....................

Utuanua Itilt 1 Stream.

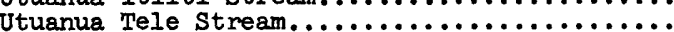

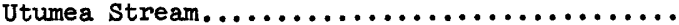

Utumoa pipeline diversion................

Utumoa stream........................

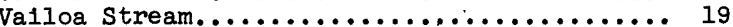

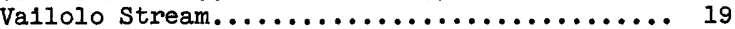

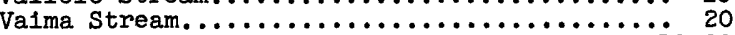

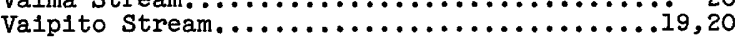

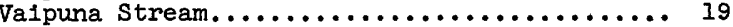

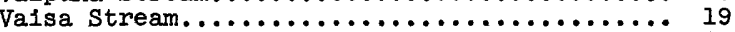

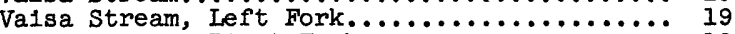

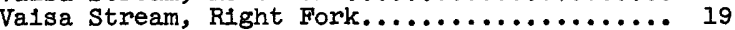

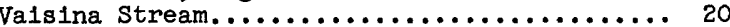

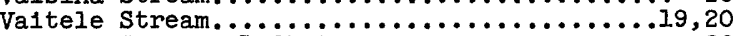

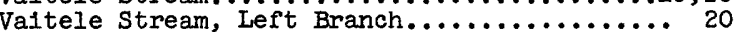

Vaitele Stream, Right Branch.............. 20

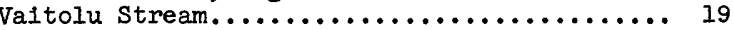

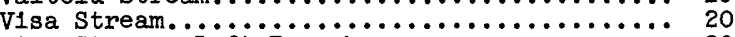

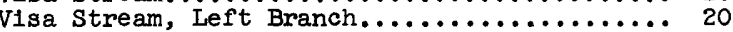

Visa Stream, Middle Fork................ 20

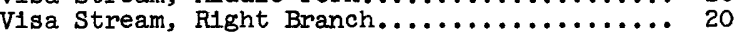

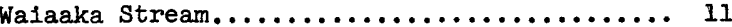

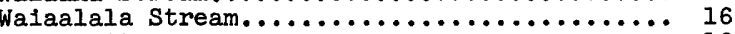

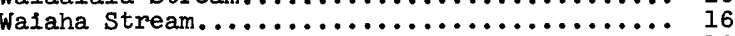

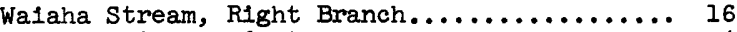

Walahi-Kuia aqueduct................... 4

Walahole Stream......................... 9

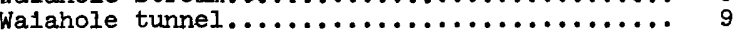

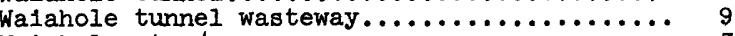

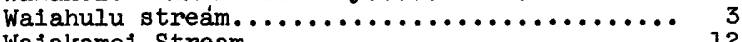

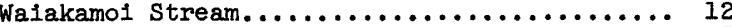

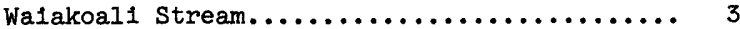

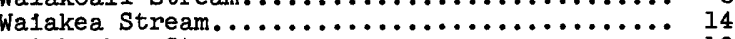

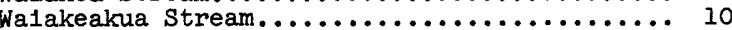

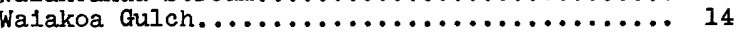

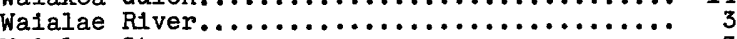

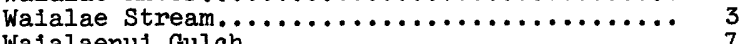

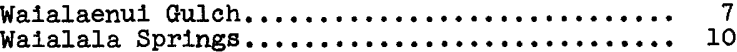

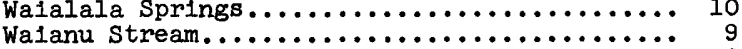

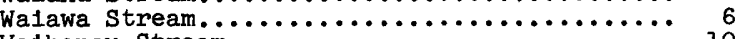

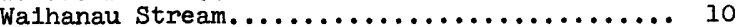

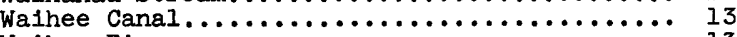

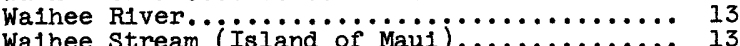

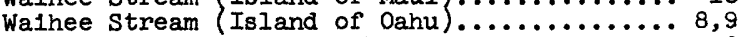

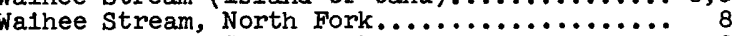

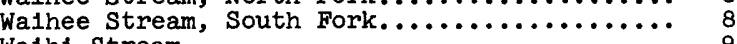

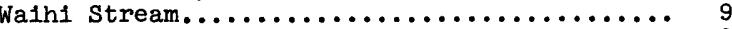

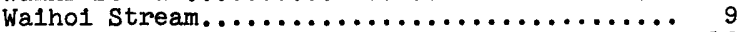

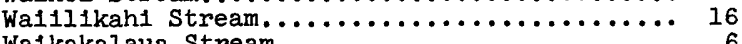

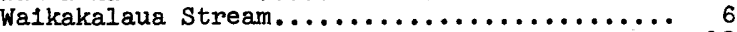

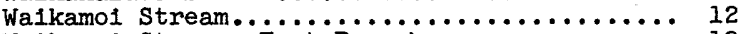

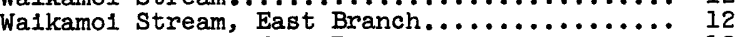

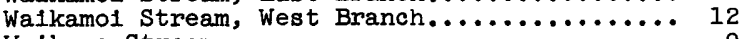

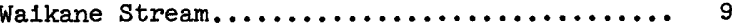

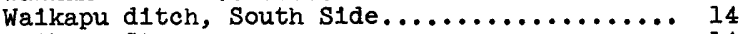

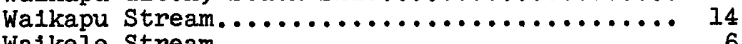

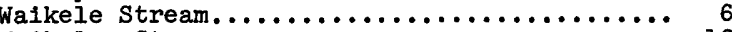

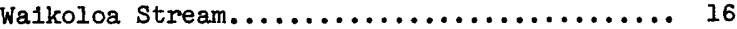

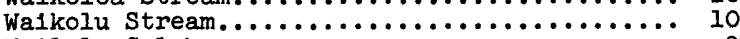

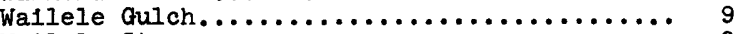

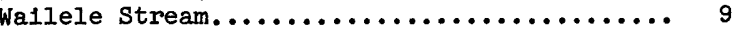

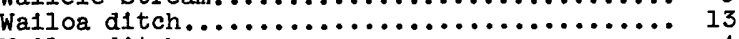

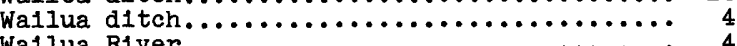

Wallua River, East Branch of Nörth Förk........

Wailua River, North Fork................ 4

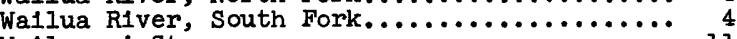

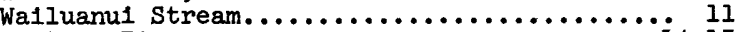

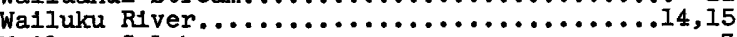

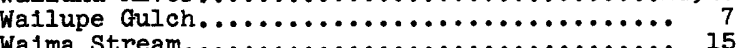

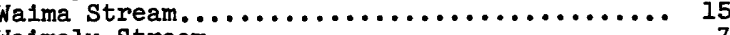

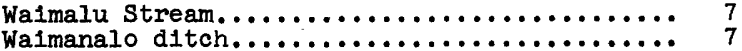

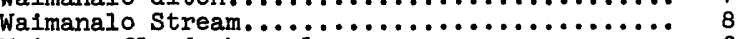

Walmano flood channel................... 6

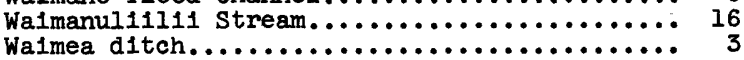


Waimea Gulch

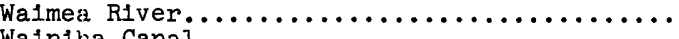

Wainiha Canal.......................

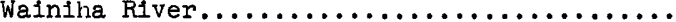

Walohinu Springs, makal station...........

Waiohinu springs, mauka station............

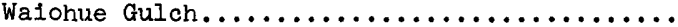

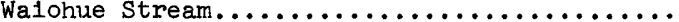

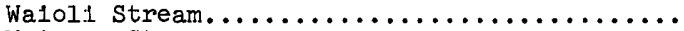

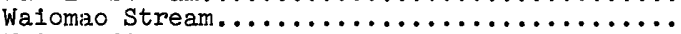

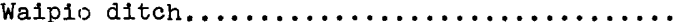

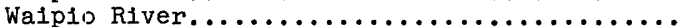

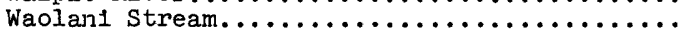

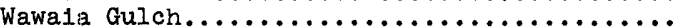

9 West Branch or Fork. See name of main stream.

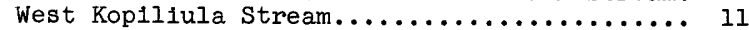

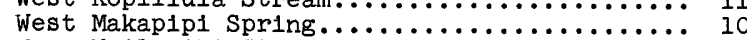

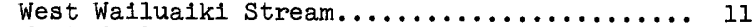

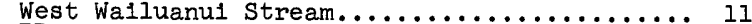

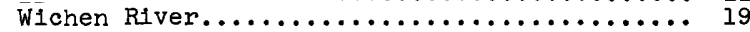
Wing Wo Ta1 ditch.................... 8

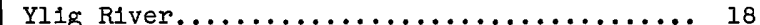

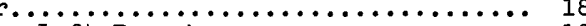

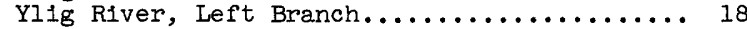

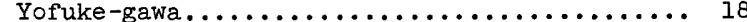

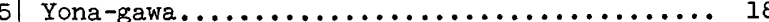

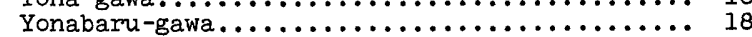

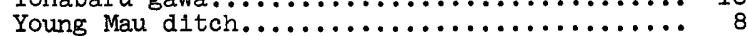


